\title{
RECOGNITION OF LINEAR ACTIONS ON SPHERES
}

\author{
BY
}

\author{
SÖREN ILLMAN
}

\begin{abstract}
Let $G$ be a finite group acting smoothly on a homotopy sphere $\Sigma^{m}$. We wish to establish necessary and sufficient conditions for the given $G$-action on $\Sigma$ to be topologically equivalent to a linear action. That is, we want to be able to decide whether or not there exists a $G$-homeomorphism $\gamma: \Sigma \rightarrow S^{m}(\rho)$, where $S^{m}(\rho) \subset$ $\mathbf{R}^{m+1}(\rho)$ denotes the unit sphere in an orthogonal representation space $\mathbf{R}^{m+1}(\rho)$ for $G$. In order for a $G$-action on $\Sigma$ to be topologically equivalent to a linear action it is clearly necessary that:

(i) For each subgroup $H$ of $G$ the fixed-point set $\Sigma^{H}$ is homeomorphic to a sphere, or empty.

(ii) For any subgroups $H$ and $H \subsetneq H_{i}, 1 \leqslant i \leqslant k$, of $G$ the pair $\left(\Sigma^{H}, \cup_{i=1}^{k} \Sigma^{H_{i}}\right)$ is homeomorphic to a standard pair $\left(S^{n}, \cup_{i=1}^{k} S_{i}^{n_{i}}\right)$, where each $S_{i}^{n_{i}}, 1 \leqslant i \leqslant k$, is a standard $n_{i}$-subsphere of $S^{n}$.

In this paper we consider the case where the fixed-point set $\Sigma^{G}$ is nonempty and all other fixed-point sets have dimension at least 5. In giving efficient sufficient conditions we do not need the full strength of condition (ii). We only need:

(ii)* For any subgroups $H$ and $H \subset H_{i}, 1 \leqslant i \leqslant p$, of $G$ such that $\operatorname{dim} \Sigma^{H_{i}}=$ $\operatorname{dim} \Sigma^{H}-2$, the pair $\left(\Sigma^{H}, \cup_{i=1}^{p} \Sigma^{\vec{H}_{i}}\right)$ is homeomorphic to a standard pair $\left(S^{n}, \cup_{i=1}^{p} S_{i}^{n-2}\right)$, where each $S_{i}^{n-2}, 1 \leqslant i \leqslant p$, is a standard $(n-2)$-subsphere of $S^{n}$

Our main results are then that, in the case when $G$ is abelian, conditions (i) and (ii)* are necessary and sufficient for a given $G$-action on $\Sigma$ to be topologically equivalent to a linear action, and in the case of an action of an arbitrary finite group the same holds under the additional assumption that any simultaneous codimension 1 and 2 fixed-point situation is simple. Our results generalize, for actions of finite groups, a well-known theorem of Connell, Montgomery and Yang, and are the first to also cover the case where codimension 2 fixed-point situations occur.
\end{abstract}

Let $G$ be a finite group acting smoothly on a homotopy sphere $\Sigma^{m}$. (A homotopy sphere $\Sigma^{m}$ is a smooth manifold which is homeomorphic to $S^{m}$.) We wish to establish necessary and sufficient conditions for the given $G$-action on $\Sigma$ to be topologically equivalent to a linear action. That is, we want to be able to decide whether or not there exists a $G$-homeomorphism $\gamma: \Sigma \rightarrow S^{m}(\rho)$, where $S^{m}(\rho) \subset$ $\mathbf{R}^{m+1}(\rho)$ denotes the unit sphere in an orthogonal representation space $\mathbf{R}^{m+1}(\rho)$ for $G$. In order for a given $G$-action on $\Sigma$ to be topologically equivalent to a linear action it is clearly necessary that the following two conditions hold:

(i) For each subgroup $H$ of $G$ the fixed-point set $\Sigma^{H}$ is homeomorphic to a sphere, or empty.

Received by the editors January 7, 1981.

1980 Mathematics Subject Classification. Primary 57S17, 57S25; Secondary 57Q30.

Key words and phrases. Smooth actions on spheres, linear actions on spheres, equivariant engulfing.

(C)1982 American Mathematical Society 0002-9947/82/0000-0358/\$07.00 
(ii) For any subgroups $H$ and $H \subset H_{i}(1 \leqslant i \leqslant k)$ of $G$ the pair $\left(\Sigma^{H}, \cup_{i=1}^{k} \Sigma^{H_{i}}\right)$ is homeomorphic to a standard pair $\left(S^{n}, \cup_{i=1}^{k} S_{i}^{n_{i}}\right)$, where each $S_{i}^{n_{i}}(1 \leqslant i \leqslant k)$ is a standard $n_{i}$-subsphere of $S^{n}$.

By a standard $q$-subsphere of $S^{n}(-1 \leqslant q \leqslant n)$, we mean an intersection of $S^{n} \subset \mathbf{R}^{n+1}$ with a $(q+1)$-dimensional linear subspace of $\mathbf{R}^{n+1}$. We also express condition (ii) by saying that the pair $\left(\Sigma^{H}, \cup_{i=1}^{k} \Sigma^{H_{i}}\right)$ is topologically standard or that $\cup_{i=1}^{k} \Sigma^{H_{i}}$ is topologically standard in $\Sigma^{H}$.

In this paper we consider the case where the fixed-point set $\Sigma^{G}$ is nonempty and all other fixed-point sets have dimension at least 5. In formulating convenient sufficient (and necessary) conditions for a given smooth $G$-action on $\Sigma$ to be topologically equivalent to a linear action we do not need the full strength of condition (ii). It is enough to assume that (ii) holds in the cases where $H_{1}, \ldots, H_{k}$ are all the subgroups for which $\operatorname{dim} \Sigma^{H_{i}}=\operatorname{dim} \Sigma^{H}-2$ and $H \underset{十}{\subset} H_{i}, i=1, \ldots, k$. Before giving our results we introduce some notation and terminology.

Let $M$ be a smooth $G$-manifold and $H$ a subgroup of $G$. Then $M^{H}$ denotes the fixed-point set of $H$ and we define

$$
M^{(>H, j)}=\bigcup_{H^{\prime} \in J(j)} M^{H^{\prime}}, \quad j=1,2,
$$

where $J(j)$ denotes the set of all subgroups $H^{\prime}$ of $G$ such that $H \subset H^{\prime}$ and $\operatorname{dim} M^{H^{\prime}}=\operatorname{dim} M^{H}-j$. (Of course $M^{(>H, j)}$ can also be defined for other values of $j$, but we only need the cases $j=1$ and 2.) We say that $M^{H}$ contains codimension $j$ fixed-point subsets if $M^{(>H, j)} \neq \varnothing(j=1,2)$, and in this case we call the pair $\left(M^{H}, M^{(>H, j)}\right)$ a codimension $j$ fixed-point situation. A codimension $j$ fixed-point situation $\left(M^{H}, M^{(>H, j)}\right)$ is simple, if there exists a subgroup $H_{j} \supsetneq H$ such that $M^{(>H, j)}=M^{H_{j}}$. If both $M^{(>H, 1)} \neq \varnothing$ and $M^{(>H, 2)} \neq \varnothing$ we call $\left(M^{H} ; M^{(>H, 1)}, M^{(>H, 2)}\right)$ a simultaneous codimension 1 and 2 fixed-point situation. (Observe that in general $M^{(>H, 2)} \nsubseteq M^{(>H, 1)}$.) A simultaneous codimension 1 and 2 fixed-point situation $\left(M^{H} ; M^{(>H, 1)}, M^{(>H, 2)}\right)$ is simple if there exist subgroups $H_{1}$ and $H_{2}$ such that $M^{(>H, 1)}=M^{H_{1}}$ and $M^{(>H, 2)}=M^{H_{2}}$. Finally, we say that a subgroup $H$ occurs in $M$ if there exists $x \in M$ such that $G_{x}=H$, where $G_{x}$ denotes the isotropy subgroup at $x$.

In the case of an action of an arbitrary finite group our main result is:

THEOREM A. Let $G$ be a finite group acting smoothly on a homotopy sphere $\Sigma$ such that $\Sigma^{G} \neq \varnothing$ and $\operatorname{dim} \Sigma^{H} \geqslant 5$ for every subgroup $H \neq G$ occurring in $\Sigma$, and such that any simultaneous codimension 1 and 2 fixed-point situation is simple. Then the action of $G$ on $\Sigma$ is topologically equivalent to a linear action if and only if the following two conditions hold:

(1) Each fixed-point set $\Sigma^{H}$ is homeomorphic to a sphere.

(2) Each codimension 2 fixed-point situation $\left(\Sigma^{H}, \Sigma^{(>H, 2)}\right)$ is topologically standard.

In the case when $G$ is abelian we are able to get rid of the extra assumption that if simultaneous codimension 1 and 2 fixed-point situations occur they must be simple. 
We prove:

THEOREM B. Let $G$ be a finite abelian group acting smoothly on $\Sigma$ such that $\Sigma^{G} \neq \varnothing$ and $\operatorname{dim} \Sigma^{H} \geqslant 5$ for every subgroup $H \neq G$ occurring in $\Sigma$. Then the action of $G$ is topologically equivalent to a linear action if and only if conditions (1) and (2) in Theorem A are satisfied.

Addendum. In the case of a simple codimension 2 fixed-point situation $\left(\Sigma^{H}, \Sigma^{(>H, 2)}\right)=\left(\Sigma^{H}, \Sigma^{H_{2}}\right)$ the condition, in Theorems A and $\mathrm{B}$, that $\left(\Sigma^{H}, \Sigma^{H_{2}}\right)$ is topologically standard can be replaced by the condition:

$$
\left\{\begin{array}{l}
\pi_{1}\left(\Sigma^{H}-\Sigma^{H_{2}}\right) \cong Z, \\
\pi_{p}\left(\Sigma^{H}-\Sigma^{H_{2}}\right)=0, \quad \text { for all } p \geqslant 2 .
\end{array}\right.
$$

Proof of Addendum. Condition (*) gives a map $\alpha$ : $S^{1} \rightarrow \Sigma^{H}-\Sigma^{H_{2}}$ which induces an isomorphism in homotopy in all dimensions. Because $\Sigma^{H}-\Sigma^{H_{2}}$ is homeomorphic to an open subset of a sphere it can be triangulated, and hence it follows that $\alpha$ is a homotopy equivalence. Since $\operatorname{dim} \Sigma^{H} \geqslant 5$ it now follows by Corollary 9.3 in Stallings [17] that $\Sigma^{H_{2}}$ is topologically standard in $\Sigma^{H}$. Conversely if $\Sigma^{H_{2}}$ is topologically standard in $\Sigma^{H}$ condition $(*)$ clearly holds.

A well-known result of Connell, Montgomery and Yang from 1964 says that if a compact Lie group $G$ acts smoothly on $S^{m}$ such that the fixed-point set $\left(S^{m}\right)^{G}$ is diffeomorphic to $S^{k}$, where $0 \leqslant k \leqslant m-3$, and all other orbits are of the same type and dimension $r$, where $m-r \geqslant 5$, then the action of $G$ is topologically equivalent to a linear action of $G$ on $S^{m}$. In fact, this result of Connell, Montgomery and Yang is a corollary to their corresponding result concerning actions on euclidean space, where the conclusion moreover is "differentiably equivalent to a linear action". (See Connell-Montgomery-Yang [5 and 6], and cf. also Siebenmann [14]). It has been a problem to generalize the Connell-Montgomery-Yang result to the case where there are more than two orbit types and fixed-point sets of codimension 2 occur, cf. Problem C.2 in [2]. A generalization to the case with several orbit types but still assuming that there are no codimension 2 fixed-point situations is given, without proof, in Rothenberg [12, Theorem 3.14 and Corollary 3.15]. Our results, for actions of finite groups, are the first to also cover the case where codimension 2 fixed-point situations occur.

It is the codimension 2 fixed-point situations that are the critical ones and give the main problems. In fact, by Giffen [7], there exist smooth actions on spheres with simple codimension 2 fixed-point situations that are not topologically standard. Giffen shows, for example, that any finite cyclic group $Z_{n}, n \geqslant 2$, can act smoothly on $S^{5}$ such that $\left(S^{5}\right)^{Z_{n}} \cong S^{3}$ is knotted in $S^{5}$. Such an action cannot be topologically equivalent to a linear action.

Let $\left(\Sigma^{H}, \Sigma^{(>H, 2)}\right)$ be a codimension 2 fixed-point situation, where $\Sigma^{(>H, 2)}=$ $\cup_{i=1}^{r} \Sigma^{H_{i}}$. Assume that each $\Sigma^{H_{i}}, 1 \leqslant i \leqslant r$, is topologically standard in $\Sigma^{H}$. We do not know whether or not it follows from this assumption that $\Sigma^{(>H, 2)}$ must be topologically standard in $\Sigma^{H}$ (cf. the formulation of Problem C.2 in [2]). 
We shall now give a very brief outline of the proof of our main results and of the contents of this paper. In $\$ 1$ we explain our notation and terminology and also introduce some preliminary facts that are used (often without specific reference) later on in the paper. In $\S 2$ we prove an equivariant engulfing theorem, Theorem 2.4, which is an equivariant analogue of Stallings' engulfing theorem [16]. The main result in $\S 3$ is Proposition 3.2 which gives necessary and sufficient conditions for an equivariant p.1. manifold $M$ (for the definition see $\$ 1$ below) to be $G$-homeomorphic to some euclidean representation space $\mathbf{R}^{m}(\rho)$. The proof of Proposition 3.2 uses the equivariant engulfing theorem and also Proposition 3.4, which is an equivariant version of the theorem of Morton Brown [3] on the monotone union of open cells. Proposition 3.2 will be applied to $M=\Sigma-\{b\}$, where $b \in \Sigma^{G} \neq \varnothing$. It is in $\S 4$ that we establish the main technical results that allow us to handle codimension 2 fixed-point situations and the interference of codimension 1 and codimension 2 fixed-point situations. The results in $\S 4$ are to be applied to the smooth $G$-manifold $M=\Sigma-\{b\}$. Lemma 4.3 is related to the case of a simple simultaneous codimension 1 and 2 fixed-point situation and is needed for the proof of Theorem A. Lemma 4.4 deals with the interference of codimension 1 and codimension 2 fixed-point situations in the case when $G$ is abelian and is a step in the proof of Theorem B. (In case Lemma 4.4 could be established for an arbitrary finite group, we would obtain the result in Theorem B for all finite groups.)

The next result, Lemma 4.5 , is the key to the handling of codimension 2 fixed-point situations, in the case of both Theorems A and B. Having established Lemmas 4.1-4.4, this Lemma 4.5 now shows that from the assumption of a certain homotopical well-behavior of the codimension 2 fixed-point situations follows a certain homotopical well-behavior of the whole situation (for a precise statement see Lemma 4.5). Finally in 5.1 we give the concluding part of the proofs of Theorems A and B. Here there are two facts that must be verified. First it is shown that the assumption that each codimension 2 fixed-point situation is topologically standard implies the homotopical well-behavior of the codimension 2 fixed-point situations needed for an application of Lemma 4.5. Then Lemma 4.5 implies the homotopical well-behavior of the whole situation, and this fact is needed in order for us to be able to apply Proposition 3.2. The other fact that we need to know, in order to be able to apply Proposition 3.2, is that $M$ is equivariantly unraveled at infinity (see Definition 3.1). The second thing shown in 5.1 is exactly this fact that $M$ is equivariantly unraveled at infinity. This property of $M=\Sigma-\{b\}$ is a consequence of the fact that we are dealing with a smooth action on $\Sigma$. We have then shown that $M$ satisfies the conditions in the assumption of Proposition 3.2 and hence by Proposition 3.2 we have that $M$ is $G$-homeomorphic to some euclidean representation space $\mathbf{R}^{m}(\rho)$. Since $\Sigma$ is the one-point compactification of $M$, it now follows that $\Sigma$ is $G$-homeomorphic to $S^{m}(\rho \oplus$ id $)$.

The equivariant engulfing theorem, Theorem 2.4, and a less general and weaker form of Theorem A were announced in [11].

1. Notation, terminology and other preliminaries. In the rest of this paper $G$ will always be a finite group. If $X$ is a $G$-space and $H$ is a subgroup of $G$, then $X^{H}$ denotes the fixed-point set of $H$, and by $X^{>H}$ we denote the set of points with 
isotropy subgroup strictly greater than $H$, i.e., $X^{>H}=\left\{x \in X \mid H \subset G_{x}\right\}$. Furthermore we denote

$$
X_{H}=X^{H}-X^{>H} \text {. }
$$

Thus $X_{H}$ is the set of points in $X$ with isotropy subgroup equal to $H$. We say that a subgroup $H$ of $G$ occurs in $X$ if there exists $x \in X$ with $G_{x}=H$, i.e., $H$ occurs in $X$ if and only if $X_{H} \neq \varnothing$.

In $\S \S 2$ and 3 we will use some equivariant p.l. techniques. The definition of an equivariant simplicial complex, and equivariant p.l. manifold, and other related matters can already be found in [9 and 10], but for the convenience of the reader they will be repeated here. In order to prevent misunderstandings the reader should pay attention to all these definitions. By a simplicial complex we mean a geometric simplicial complex, that is, the topological realization of an abstract simplicial complex considered as a topological space together with the structure given by the simplexes. In this paper all simplicial complexes will be countable, locally finite and finite-dimensional. A simplicial $G$-complex consists of a simplicial complex $K$ together with a $G$-action $\varphi: G \times X \rightarrow X$ such that the map $g: X \rightarrow X$ is a simplicial homeomorphism for every $g \in G$. We define an equivariant simplicial complex to be a simplicial $G$-complex $K$ which satisfies the following two conditions.

1. For every subgroup $H$ of $G$ we have that if $s=\left\langle v_{0}, \ldots, v_{m}\right\rangle$ is a simplex of $K$ and $s^{\prime}=\left\langle h_{0} v_{0}, \ldots, h_{m} v_{m}\right\rangle$, where $h_{i} \in H, i=0, \ldots, m$, also is a simplex of $K$ then there exists $h \in H$ such that $h v_{i}=h_{i} v_{i}, i=0, \ldots, m$.

2. For any simplex $s$ of $K$ the vertices $v_{0}, \ldots, v_{n}$ of $s$ can be ordered in such a way that we have $G_{v_{n}} \subset \cdots \subset G_{v_{0}}$.

In condition 1 the vertices $v_{0}, \ldots, v_{m}$ need not be distinct. We call $G_{v_{n}}$ the principal isotropy subgroup of the simplex $s$ and $G_{v_{0}}$ the maximal isotropy subgroup of $s$. The above conditions are purely technical since the second barycentric subdivision of any simplicial $G$-complex is an equivariant simplicial complex. (An abstract simplicial $G$-complex satisfying condition 1 above is called regular by Bredon [1, p. 116].) Moreover it is easy to see that in the presence of condition 2 condition 1 can be replaced by the following.

$1^{\prime}$. If $s=\left\langle v_{0}, \ldots, v_{m}\right\rangle$ is a simplex of $K$ and $s^{\prime}=\left\langle g_{0} v_{0}, \ldots, g_{m} v_{m}\right\rangle$, where $g_{i} \in G$, $i=1, \ldots, m$, also is a simplex of $K$ then there exists $g \in G$ such that $g v_{i}=g_{i} v_{i}$, $i=0, \ldots, m$.

Condition 1 itself is a stronger condition than $1^{\prime}$, but a simplicial $G$-complex satisfies conditions 1 and 2 if and only if it satisfies conditions $1^{\prime}$ and 2 (see Lemma 1.1 and example (a) after Lemma 1.1 in [10]). Observe that in particular an equivariant simplicial complex $K$ satisfies the following. For any simplex $s$ of $K$ and any $g \in G$ we have that if $g(s)=s$ then $g y=y$ for all $y \in s$. For any simplex $s$ of $K$ we have that if $x \in s$ and $g x \in s$ then $g x=x$, and moreover if $x \in s$ and $g x=x$ then $g y=y$ for every $y \in s$. If $s=\left\langle v_{0}, \ldots, v_{n}\right\rangle$, where $G_{v_{n}} \subset \cdots \subset G_{v_{0}}$, is an $n$-simplex of the equivariant simplicial complex $K$ then every point $y$ in the set $\left\langle v_{0}, \ldots, v_{i}\right\rangle-\left\langle v_{0}, \ldots, v_{i-1}\right\rangle, 0 \leqslant i \leqslant n$, has isotropy subgroup equal to $G_{i}$. Observe in particular that for the principal isotropy subgroup $H=G_{v_{n}}$ of $s$ we have $H x=x$ for all $x \in s$. 
The orbit space $K / G$ of an equivariant simplicial complex $K$ has the structure of an ordinary simplicial complex such that the natural projection $p: K \rightarrow K / G$ is simplicial and $p$ maps each simplex of $K$ homeomorphically onto the corresponding image simplex of $K / G$.

If $\mathbf{R}^{m}(\rho)$ is an orthogonal representation space for $G$ we denote

$$
D^{m}(\rho)=\text { convex hull of }\left\{ \pm g e_{i} \mid g \in G, i=1, \ldots, m\right\},
$$

and $\partial D^{m}(\rho)$ denotes the boundary of $D^{m}(\rho)$. Here $e_{1}, \ldots, e_{m}$ denote the standard unit vectors in $\mathbf{R}^{m}$. (In [9 and 10] we have denoted $S^{m-1}(\rho)=\partial D^{m}(\rho)$, but in this paper we will reserve the notation $S^{m-1}(\rho)$ for the round $G$-sphere $S^{m-1}(\rho)=\{x \in$ $\left.\mathbf{R}^{m}(\rho) \mid\|x\|=1\right\}$.) Both $\partial D^{m-1}(\rho)$ and $D^{m}(\rho)$ can be given the structure of rectilinear equivariant simplicial complexes in $\mathbf{R}^{m}(\rho)$ (see $[9$, p. 205]), and any two rectilinear equivariant simplicial complexes $K_{1}$ and $K_{2}$ in $\mathbf{R}^{m}(\rho)$ with $\left|K_{1}\right|=\left|K_{2}\right|=$ $\partial D^{m}(\rho)\left(\right.$ or $\left.=D^{m}(\rho)\right)$ have a common equivariant simplicial subdivision (see $[9, \mathrm{pp}$. 202-203]).

An equivariant simplicial complex $K$ is an $m$-dimensional equivariant combinatorial manifold (without boundary) if for every vertex $v$ of $K$ there exists an orthogonal representation $\rho_{v}: G_{v} \rightarrow O(m)$ and a p.1. $G_{v}$-homeomorphism

$$
\alpha: \operatorname{Lk}(v, K) \rightarrow \partial D^{m}\left(\rho_{v}\right) .
$$

Let $S$ be a $G$-space. An equivariant triangulation of $S$ consists of an equivariant simplicial complex $K$ and a $G$-homeomorphism $t: K \rightarrow S$. Two equivariant triangulations $t_{1}: K_{1} \rightarrow S$ and $t_{2}: K_{2} \rightarrow S$ are called equivalent if $t_{2}^{-1} t_{1}: K_{1} \rightarrow K_{2}$ is a p.1. $G$-homeomorphism. Given an equivariant triangulation $t$ we have the class of all equivariant triangulations of $S$ equivalent to $t$, called the preferred class of equivariant triangulations of $S$ determined by $t$. Two equivariant triangulations $t_{1}$ and $t_{2}$ of $S$ determine the same class of preferred triangulations of $S$ if and only if $t_{1}$ and $t_{2}$ are equivalent. An equivariant polyhedron $P$ is a $G$-space $P$ together with a (nonempty!) preferred class of equivariant triangulations of $P$. An equivariant subpolyhedron $Q$ of $P$ is a $G$-subset of $P$ such that for some preferred equivariant triangulation $h: K \rightarrow P$ there exists an equivariant subcomplex $L$ of $K$ such that $h(L)=Q$. In particular it follows that $Q$ is a closed subset of $P$. An $m$-dimensional equivariant p.l. manifold $M$ is an equivariant polyhedron such that in the class of preferred equivariant triangulations of $M$ there exists an equivariant triangulation $t: K \rightarrow M$ with $K$ an $m$-dimensional equivariant combinatorial manifold. In fact, in this case, for any preferred equivariant triangulation $t^{\prime}: L \rightarrow M$ we have that $L$ is an $m$-dimensional equivariant combinatorial manifold.

Observe that if $M$ is an equivariant p.1. manifold then $M^{H}$ is a (locally flat) p.1. submanifold of $M$ for every subgroup $H$ of $G$. If $M$ is an equivariant p.1. manifold we use $M_{H}^{\alpha}$ to denote a component of $M_{H}$ and $M_{\beta}^{H}$ to denote a component of $M^{H}$. Observe that if $M_{\beta}^{H}$ is the component of $M^{H}$ containing $M_{H}^{\alpha}$ then $\operatorname{dim} M_{\beta}^{H}=\operatorname{dim} M_{H}^{\alpha}$, since $M$ is an equivariant p.1. manifold. If $K$ is a $G$-subset of $M$ we often denote $\left[K_{H}\right]^{\alpha}=K_{H} \cap M_{H}^{\alpha}$ and $\left[K_{H}\right]_{\beta}=K_{H} \cap M_{\beta}^{H}$.

We will often use the fact that equivariant p.1. manifolds, as well as smooth $G$-manifolds, have the property given by the following lemma. 
LEMMA. Let $M$ be an equivariant p.l. manifold (or a smooth $G$-manifold), and let $H$ be a subgroup of $G$ such that $M^{H}$ is connected. Then there exists a subgroup $\bar{H}$ of $G$, with $H \subset \bar{H}$, such that $M^{\bar{H}}=M^{H}$ and $\bar{H}$ occurs in $M$. Moreover $\bar{H}$ is uniquely determined, and if $W$ is any nonempty open subset of $M^{H}$ there exists $x \in W$ with $G_{x}=\bar{H}$.

Proof. That smooth $G$-manifolds have this property is a well-known fact and the argument for the case of equivariant p.1. manifolds is the same, i.e., it runs as follows: Let $\delta$ denote the family of all subgroups $H^{\prime}$ of $G$ such that $H \subset H^{\prime}$ and $\operatorname{dim} M^{H^{\prime}}=\operatorname{dim} M^{H}=n$. Then $\mathcal{S}$ is nonempty and if $H^{\prime} \in \mathcal{S}$ we have $M^{H^{\prime}} \subset M^{H}$. Let $\left(M^{H^{\prime}}\right)_{0}$ denote a component of $M^{H^{\prime}}$ such that $\operatorname{dim}\left(M^{H^{\prime}}\right)_{0}=n$. Since $\left(M^{H^{\prime}}\right)_{0}$ is a connected submanifold of $M^{H}$ of the same dimension as $M^{H}$ we have that $\left(M^{H^{\prime}}\right)_{0}$ is open in $M^{H}$. Since $\left(M^{H^{\prime}}\right)_{0}$ is closed in $M^{\prime}$ and $M^{\prime}$ is closed in $M^{H}$ we have that $\left(M^{H^{\prime}}\right)_{0}$ is closed in $M^{H}$. Thus $\left(M^{H^{\prime}}\right)_{0}$ is both open and closed in $M^{H}$ and hence $\left(M^{H^{\prime}}\right)_{0}=M^{H}$, since $M^{H}$ is connected. Because $M^{H^{\prime}} \subset M^{H}$ it now follows that $M^{H^{\prime}}=M^{H}$. We have shown that $H^{\prime} \in \mathcal{\delta}$ implies that $M^{H^{\prime}}=M^{H}$. Therefore it follows that if $H^{\prime}, H^{\prime \prime} \in \mathcal{S}$ then also $\left\langle H^{\prime}, H^{\prime \prime}\right\rangle \in \mathcal{S}$, where $\left\langle H^{\prime}, H^{\prime \prime}\right\rangle$ denotes the subgroup of $G$ generated by $H^{\prime}$ and $H^{\prime \prime}$. Hence there exists a largest subgroup $\bar{H}$ in S.

We now claim that if $W$ is any nonempty open subset of $M^{H}$ there exists $x \in W$ such that $G_{x}=\bar{H}$. This is seen as follows. Let $\tilde{H}$ be any subgroup such that $\bar{H} \subset \tilde{H}$. By the maximality of $\bar{H}$ we have $\tilde{H} \notin \mathcal{S}$, and since $H \subset \tilde{H}$ we must then have $\operatorname{dim} M^{\tilde{H}}<n$. If $n=0$ we have $M^{H}=\{a\}$, and then also $M^{\bar{H}}=\{a\}$ and $W=\{a\}$. Since $M^{\tilde{H}}=\varnothing$, we have $G_{a}=\bar{H}$. Now assume $n>0$. Give $M$ an equivariant triangulation, which we again denote by $M$, such that some vertex $v$ of $M^{\bar{H}}$ lies in $W$. Let $\alpha: \operatorname{St}(v, M) \rightarrow D^{m}\left(\rho_{v}\right)$ be a p.1. $G_{v}$-homeomorphism, where $\rho_{v}: G_{v} \rightarrow O(m)$. We have $\bar{H} \subset G_{v}$ and $\operatorname{dim}\left[\mathbf{R}^{m}\left(\rho_{v}\right)\right]^{\bar{H}}=n$. Since for any subgroup $\tilde{H}$ such that $\bar{H} \subset \tilde{H}$ we have $\operatorname{dim} M^{\tilde{H}}<n$ it follows that $\left[\mathbf{R}^{m}\left(\rho_{v}\right)\right]^{>\bar{H}}$ equals a finite union of linear subspaces of dimension $\leqslant n-1$. Hence $W^{\prime} \cap\left[\mathbf{R}^{m}\left(\rho_{v}\right)\right]_{\bar{H}} \neq \varnothing$, for any neighbourhood $W^{\prime}$ of the origin in $\left[\mathbf{R}^{m}\left(\rho_{v}\right)\right]^{H}$, and therefore $W \cap \operatorname{St}(v, M)_{\bar{H}} \neq \varnothing$. Any $x \in W \cap$ $\operatorname{St}(v, M)_{\bar{H}}$ then satisfies $G_{x}=\bar{H}$ and $x \in W$.

To see that $\bar{H}$ is uniquely determined let $\bar{H}$ and $\overline{\bar{H}}$ be two subgroups of $G$ such that $M^{\bar{H}}=M^{H}=M^{\bar{H}}$, and both $\bar{H}$ and $\overline{\bar{H}}$ occur in $M$. Let $x \in M$ be such that $G_{x}=\bar{H}$. Then $x \in M^{\bar{H}} \cap M^{\bar{H}}=M^{\langle\bar{H}, \bar{H}\rangle}$, where $\langle\bar{H}, \overline{\bar{H}}\rangle$ denotes the subgroup of $G$ generated by $\bar{H}$ and $\overline{\bar{H}}$. Thus $G_{x} \supset\langle\bar{H}, \overline{\bar{H}}\rangle \supset \bar{H}$, and hence $\langle\bar{H}, \overline{\bar{H}}\rangle=\bar{H}$. Likewise $\langle\bar{H}, \overline{\bar{H}}\rangle=\overline{\bar{H}}$, and hence $\bar{H}=\overline{\bar{H}}_{x}$.

Let us also here give a remark, that will be used in 5.1, on the notion of $\Sigma^{(>H, 2)}$ being topologically standard in $\Sigma^{H}$. Recall from the introduction that $\Sigma^{(>H, 2)}$ is topologically standard in $\Sigma^{H}$ means that there exists a homeomorphism $\alpha: \Sigma^{H} \rightarrow S^{n}$ such that $\alpha\left(\Sigma^{(>H, 2)}\right)$ equals a union of standard $(n-2)$-dimensional subspheres of $S^{n}$. Let $H_{1}, \ldots, H_{r}$ be such that $\Sigma^{(>H, 2)}=\cup_{i=1}^{r} \Sigma^{H_{i}}$. Now let $\bar{L}_{i}, 1 \leqslant i \leqslant r$, be (n-- 1)-dimensional linear subspaces of $\mathbf{R}^{n+1}$ such that $\alpha\left(\Sigma^{H_{i}}\right)=S^{n} \cap \bar{L}_{i}$. By our assumption $\Sigma^{G}$ is homeomorphic to a nonempty sphere and hence $\Sigma^{G}$ contains at least two points. Let $a, b \in \Sigma^{G}$, where $a \neq b$. We have $\bar{b}=\alpha(b) \in \cap_{i=1}^{r} \alpha\left(\Sigma^{H_{i}}\right)$, 
and hence in particular $0 \neq \bar{b} \in \bar{L}_{0}=\bigcap_{i=1}^{r} \bar{L}_{i}$. Thus $\bar{L}_{0}$ is a linear subspace of $\mathbf{R}^{n+1}$ with $\operatorname{dim} \bar{L}_{0} \geqslant 1$. Choose coordinates in $\mathbf{R}^{n+1}$ such that $\bar{b}=(0, \ldots, 0,1)$ and write $\mathbf{R}^{n+1}=\mathbf{R}^{n} \oplus \mathbf{R} \bar{b}$. Since $\mathbf{R} \bar{b} \subset \bar{L}_{0} \subset \bar{L}_{i}$, for $1 \leqslant i \leqslant r$, it follows that each $\bar{L}_{i}$ is of the form $\bar{L}_{i}=L_{i} \oplus \mathbf{R} \bar{b}$, where $L_{i}$ is an $(n-2)$-dimensional linear subspace of $\mathbf{R}^{n}$. Now define a homeomorphism $\gamma: S^{n}-\{\bar{b}\} \rightarrow \mathbf{R}^{n}$ by

$$
\gamma\left(x, x_{n+1}\right)= \begin{cases}x \in \mathbf{R}^{n}, & \text { if } x_{n+1} \leqslant 0, \\ \frac{x}{\|x\|^{2}}, & \text { if } 0 \leqslant x_{n+1}<1 .\end{cases}
$$

Then $\left(\left(S^{n}-\left\{b^{\prime}\right\}\right) \cap \overline{L_{i}}\right)=L_{i}$, for $1 \leqslant i \leqslant r$. Since $a^{\prime}=\gamma \alpha(a) \in L_{i}$, for all $i$, it follows that the affine map $t: \mathbf{R}^{n} \rightarrow \mathbf{R}^{n}$, defined by $t(x)=x-a^{\prime}$, satisfies $t\left(L_{i}\right)=L_{i}$, $1 \leqslant i \leqslant r$. Therefore

$$
\alpha^{\prime}=t \circ \gamma \circ \alpha: \Sigma^{H}-\{b\} \stackrel{\cong}{\stackrel{9}{\rightarrow}} \mathbf{R}^{n}
$$

is a homeomorphism with $\alpha^{\prime}(a)=0$ and such that $\alpha^{\prime}\left(\Sigma^{H_{i}}-\{b\}\right)=L_{i}$, an $(n-2)$ dimensional linear subspace of $\mathbf{R}^{n}$, for $1 \leqslant i \leqslant r$, and thus $\alpha^{\prime}\left(\Sigma^{(>H, 2)}-\{b\}\right)=$ $\cup_{i=1}^{r} L_{i}$. It is in this form that the assumption that $\Sigma^{(>H, 2)}$ is topologically standard in $\Sigma^{H}$ is put to use in the proof of 5.1.

We will occasionally deal with nonconnected manifolds. All notions we use for nonconnected manifolds have their obvious interpretation, i.e., they hold for all components of the manifolds.

Let us point out one final notation, used in the proof of 2.4. Let $s$ be a simplex with vertices $v_{0}, \ldots, v_{q}$, where $q \geqslant 1$, and let $0<\varepsilon<1 /(q+1)$. Then $s_{\varepsilon}$ denotes the smaller simplex inside $s$ given by

$$
s_{\varepsilon}=\left\{x \in s \mid x=\sum_{i=0}^{q} t_{i} v_{i}, \varepsilon \leqslant t_{i}<1, \sum_{i=0}^{q} t_{i}=1\right\} .
$$

2. The equivariant engulfing theorem. In this section we establish an equivariant analogue of Stallings' engulfing theorem [16]. First we consider equivariant collapsing and prove the easy but crucial Proposition 2.3 below.

Let $L$ be an equivariant subcomplex of the equivariant simplicial complex $K$. We say that there is an elementary $G$-simplicial collapse from $K$ to $L$ if $K-L$ consists of two equivariant simplexes $G s$ and $G s_{1}$ with $s_{1}$ a face of $s$ and such that $s_{1}$ and $s$ have the same principal isotropy subgroup and $s=v s_{1}$, where $v$ is a vertex of $s$. We say that $K$ collapses $G$-simplicially to $K_{0}$ if there is a sequence $K=K_{r} \supset K_{r-1} \supset \cdots \supset K_{0}$ of equivariant subcomplexes of $K$ such that there is an elementary $G$-simplicial collapse from $K_{i}$ to $K_{i-1}$, for each $i=1, \ldots, r$.

Let $Q$ be an equivariant subpolyhedron of the equivariant polyhedron $P$, and let $p: P \rightarrow P / G$ denote the projection onto the orbit space. We say that there is an elementary equivariant collapse (more precisely, an elementary equivariant $m$-dimensional collapse of type $(H))$ from $P$ to $Q$ if the following holds. There is a p.1. $m$-ball $B^{m}$ in $P$ such that $p \mid: B^{m} \rightarrow p\left(B^{m}\right)$ is a homeomorphism and a p.1. $(m-1)$-ball $B^{m-1} \subset \partial B^{m}$ such that $Q \cap B^{m}=B^{m-1}$ and $P=Q \cup G B^{m}$ and all points in 
$B^{m}-B^{m-1}$ have the same isotropy subgroup $H$. We say that $P$ collapses equivariantly to $P_{0}$ if there exists a sequence $P=P_{t} \supset P_{t-1} \supset \cdots \supset P_{0}$ of equivariant subpolyhedra of $P$ such that there is an elementary equivariant collapse from $P_{j}$ to $P_{j-1}$, for $j=1, \ldots, t$.

Let $s$ be an ordinary simplex of the equivariant simplicial complex $K$ and let $H$ be the principal isotropy subgroup of $s$. Then $\operatorname{st}(s ; K)$ and $\operatorname{lk}(s ; K)$ are $H$-equivariant simplicial complexes and if $g \notin H$ we have st $(s ; K) \cap s \mathrm{st}(g s ; K)=\varnothing$. Thus, if $b \in s$ and we star $s$ at $b$ and all the other simplexes $g_{1} s, \ldots, g_{k} s$ at $g_{1} b, \ldots, g_{k} b$, respectively, we get a well-defined $G$-equivariant subdivision $K^{\prime}$ of $K$. In this case we say that $K^{\prime}$ is obtained from $K$ by an elementary $G$-starring at $G b \in G s^{\circ}$. We say that $K^{*}$ is an equivariant stellar subdivision of $K$ if there exists a sequence $K, K^{\prime}, K^{\prime \prime}, \ldots, K^{(r)}=K^{*}$, where each $K^{(i)}$ is obtained from $K^{(i-1)}$ by an elementary $G$-starring, $i=1, \ldots, r$. If $K^{*}$ is an equivariant stellar subdivision of $K$ the induced subdivision $K^{*} / G$ of $K / G$ is stellar, and in fact conversely if $(K / G)^{*}$ is a stellar subdivision of $K / G$ then the induced equivariant subdivision $K^{*}$ of $K$ is an equivariant stellar subdivision of $K$.

Lemma 2.1. Assume that $K$ collapses $G$-simplicially to $K_{0}$ and let $K^{*}$ be an equivariant stellar subdivision of $K$. Then $K^{*}$ collapses $G$-simplicially to $K_{0}^{*}$.

Proof. It is enough to consider the case when there is an elementary $G$-simplicial collapse from $K$ to $K_{0}$ and where the equivariant stellar subdivision is an elementary $G$-starring. The proof is then completely analogous to the standard proof in the ordinary case, see for example Hudson [8, Lemma 2.2].

LEMma 2.2. Let $K_{0}$ be an equivariant subcomplex of the equivariant simplicial complex $K$, such that the equivariant polyhedron $|K|$ collapses equivariantly to $\left|K_{0}\right|$. Then there exists an equivariant subdivision $K^{*}$ of $K$ such that $K^{*}$ collapses $G$-simplicially to $K_{0}^{*}$.

Proof. Let us first show that if $L_{0}$ is an equivariant subcomplex of the equivariant simplicial complex $L$ such that there is an elementary equivariant collapse from $|L|$ to $\left|L_{0}\right|$, then there exists an equivariant subdivision $L^{*}$ of $L$ such that $L_{0}^{*}$ is an equivariant stellar subdivision of $L_{0}$ and such that $L^{*}$ collapses $G$-simplicially to $L_{0}^{*}$. By the assumption there is a p.1.-ball $B^{m}$ in $|L|$, such that $B^{m}$ injects into the orbit space and $B^{m} \cap\left|L_{0}\right|=B^{m-1}$ is an $(m-1)$-ball in $\partial B^{m}$ and $|L|=\left|L_{0}\right| \cup G B$, and moreover all points in $B^{m}-B^{m-1}$ have the same isotropy subgroup. Let $p:|L| \rightarrow$ $|L| / G$ denote the projection onto the orbit space. Then $p\left(B^{m}\right)$ is a p.1. $m$-ball in $|L| / G$ and $\left|L_{0}\right| / G \cap p\left(B^{m}\right)=p\left(\left|L_{0}\right| \cap B^{m}\right)=p\left(B^{m-1}\right)$ is a p.1. $(m-1)$-ball in $\partial\left(p\left(B^{m}\right)\right)$ and $|L| / G=\left|L_{0}\right| / G \cup p\left(B^{m}\right)$, i.e., there is an elementary collapse from $|L| / G=|L / G|$ to $\left|L_{0}\right| / G=\left|L_{0} / G\right|$. Hence (see e.g. Hudson [8, Lemma 2.3]) there is a subdivision $(L / G)^{*}$ of $L / G$ such that $\left(L_{0} / G\right)^{*}$ is a stellar subdivision of $L_{0} / G$ and such that $(L / G)^{*}$ collapses simplicially to $\left(L_{0} / G\right)^{*}$. Let $L^{*}$ denote the induced equivariant subdivision of $L$. Then $L_{0}^{*}$ is an equivariant stellar subdivision of $L_{0}$. Let $(L / G)^{*}=R_{q} \backslash R_{q-1} \searrow \cdots \searrow R_{0}=\left(L_{0} / G\right)^{*}$ be a sequence of elementary simplicial collapses. Since all points in $|L / G|-\left|L_{0} / G\right|$ have the same orbit 
type it follows immediately that $L^{*}=L_{q}^{*} \searrow L_{q-1}^{*} \searrow \cdots \searrow L_{0}^{*}$, where $L_{i}^{*}=p^{-1}\left(R_{i}\right)$, $i=0, \ldots, q$, is a sequence of elementary $G$-simplicial collapses.

The lemma now follows, by induction, from the above and Lemma 2.1.

Proposition 2.3. Let $M$ be an equivariant p.l. manifold without boundary and let $Q \subset P$ be equivariant subpolyhedra of $M$, such that $P$ collapses equivariantly to $Q$. Let $U$ be an open $G$-subset of $M$ such that $Q \subset U$. Then there exist a compact $G$-subset $E$ and an ambient $G$-isotopy $h_{t} \operatorname{rel}((M-E) \cup Q)$ such that $P \subset h_{1}(U)$.

Proof. Let $L \subset K \subset J$ be an equivariant triangulation of $Q \subset P \subset M$, such that $K$ collapses $G$-simplicially to $L$. Then $J$ is an equivariant combinatorial manifold. Next observe that for any $r$-simplex $\sigma$ of $J$, such that $\sigma \subset J^{H}$, we have that $\operatorname{lk}(\sigma ; J)$ is p.1. $H$-homeomorphic to a p.1. $H$-sphere $S^{m-r-1}(\rho)$, where $\mathbf{R}^{m-r}(\rho)$ is an orthogonal representation space for $H$ and $m=\operatorname{dim} M=\operatorname{dim} J$. This follows, by induction, from the fact that $\operatorname{lk}(\sigma ; J)=\operatorname{lk}\left(w ; \operatorname{lk}\left(\sigma_{1} ; J\right)\right)$, where $w$ is a vertex of $\sigma$ and $\sigma_{1}$ is the face of $\sigma$ opposite $w$.

It is enough to consider the case where there is an elementary $G$-simplicial collapse from $K$ to $L$. Let $G s_{1}$ and $G s$ be the equivariant simplexes of $K-L$, where $s_{1}$ is a face of $s$, and $s_{1}$ and $s$ have the same principal isotropy subgroup, say $H$, and $s=s_{1} v$, where $v$ is a vertex of $s$. Thus $v$ is fixed under $H$. We have $\dot{s}_{1} v \subset L \subset U$. Let $b \in s_{1}$ be the barycenter of $s_{1}$, and choose $c \in b v$, where $c \neq v$, so close to $v$ that $\dot{s}_{1} v c \subset U$.

Since $q=\operatorname{dim} s_{1} \leqslant m-1$, we have $\operatorname{lk}\left(s_{1} ; J\right) \neq \varnothing$. Let $\alpha: \operatorname{lk}\left(s_{1} ; J\right) \rightarrow S^{m-q-1}(\rho)$ be a p.1. $H$-homeomorphism where $S^{m-q-1}(\rho)$ is the p.1. $H$-sphere in the orthogonal representation space $\mathbf{R}^{m-q}(\rho)$. Since $\alpha(v)$ is fixed under $H$ we have $\operatorname{dim}\left(\mathbf{R}^{m-q}(\rho)\right)^{H}$ $\geqslant 1$. Let $V$ be the 1-dimensional linear subspace of $\mathbf{R}^{m-q}(\rho)$ generated by $\alpha(v)$. Now let $\mathbf{R}^{m-q}(\rho)=V \oplus W$, where $W$ is an $H$-invariant $(m-q-1)$-dimensional linear subspace of $\mathbf{R}^{m-q}(\rho)$. Denote $\bar{v}=\alpha(v) /\|\alpha(v)\| \in V$. There exists a p.1. H-homeomorphism $\gamma: S^{m-q-1}(\rho) \rightarrow S(V \oplus W)=\{\bar{v},-\bar{v}\} S(W)$, such that $\gamma(\alpha(v))=\bar{v}$. We denote $\beta=\gamma \alpha: \operatorname{lk}\left(s_{1} ; J\right) \rightarrow S(V \oplus W)$. Let $\bar{s}_{1} \subset \mathbf{R}^{q}$ denote a copy of $s_{1}$ linearly imbedded in $\mathbf{R}^{q}$ (with trivial $H$-action). Define

$$
\varphi=\mathrm{id} * \beta: \operatorname{st}\left(s_{1} ; J\right)=s_{1} \operatorname{lk}\left(s_{1} ; J\right) \rightarrow \bar{s}_{1} S(V \oplus W) \quad\left(\subset \mathbf{R}^{q} \oplus V \oplus W\right) .
$$

Then $\varphi$ is a p.1. $H$-homeomorphism and $\varphi(s)=\varphi\left(s_{1} v\right)=\bar{s}_{1} \bar{v}$. Let us further denote $U^{\prime}=U \cap \operatorname{st}\left(s_{1} ; J\right)$ and $\varphi(c)=\bar{c} \in \mathbf{R}^{q} \oplus V$ and $\varphi(b)=\bar{b} \in \mathbf{R}^{q}$. Then $\dot{\overline{s_{1}}} \bar{v} \bar{c} \subset \varphi\left(U^{\prime}\right)$. Now let

$$
\eta: \bar{s}_{1}\{\bar{v},-\bar{v}\} \rightarrow \bar{s}_{1}\{\bar{v},-\bar{v}\}
$$

be a p.1. homeomorphism, keeping the boundary fixed, such that $\bar{s}_{1} \bar{v} \subset \eta\left(\dot{\overline{s_{1}}} \bar{v} \bar{c}\right)$. [For example by defining $\eta(\bar{v})=\bar{v}, \eta(\bar{c})=\bar{b}, \eta(\bar{b})=-\bar{c}, \eta(-\bar{v})=-\bar{v}$ and $\eta \mid \dot{\bar{s}}_{1}=$ id and extending linearly to the corresponding simplexes.] We define

$$
\omega=\eta * \operatorname{id}: \bar{s}_{1}\{\bar{v},-\bar{v}\} S(W) \rightarrow \bar{s}_{1}\{\bar{v},-v\} S(W) .
$$

Then $\xi=\varphi^{-1} \omega \varphi: \operatorname{st}\left(s_{1} ; J\right) \rightarrow \operatorname{st}\left(s_{1} ; J\right)$ is a p.1. $H$-homeomorphism keeping the boundary fixed, such that $\xi\left(U^{\prime}\right) \supset \varphi^{-1} \omega(\dot{\bar{s}} \bar{v} \bar{c}) \supset \varphi^{-1}\left(\bar{s}_{1} \bar{v}\right)=s$. By Lemma 2.10 in [10] there is an ambient p.1. $H$-isotopy $h_{t}^{\prime}$ of $\operatorname{st}\left(s_{1} ; J\right) \operatorname{rel} \partial\left(\operatorname{st}\left(s_{1} ; J\right)\right)=\dot{s}_{1} \operatorname{lk}\left(s_{1} ; J\right)$ 
from id to $\xi$. Extending $h_{t}^{\prime}$ equivariantly we get an ambient p.1. $G$-isotopy of $G \operatorname{st}\left(s_{1} ; J\right) \operatorname{rel} G\left(\partial \operatorname{st}\left(s_{1} ; J\right)\right)$ and by extending this by the identity outside $G \operatorname{st}\left(s_{1} ; J\right)$ we get a well-defined ambient p.1. $G$-isotopy $h_{t}$ of $M=|J| \operatorname{rel}((M-E) \cup L)$, where $E=G \operatorname{st}\left(s_{1} ; J\right)$, such that $h_{1}(U) \supset|K|=P$.

We are now ready to prove the equivariant engulfing theorem.

THEOREM 2.4. Let $M$ be an equivariant p.l. manifold, without boundary. Let $U$ be an open $G$-subset of $M$ and let $L \subset K$ be equivariant subpolyhedra of $M$ such that $L \subset U$ and $\overline{K-L}$ is compact, and $\operatorname{dim}\left[K_{H}\right]^{\alpha} \leqslant \operatorname{dim} M_{H}^{\alpha}-3$, for every subgroup $H$ and component $M_{H}^{\alpha}$ of $M_{H}$ for which $\left[(K-L)_{H}\right]^{\alpha} \neq \varnothing$. Assume that $\left(M_{H},\left[U_{H}\right]^{\alpha}\right)$ is $r(H, \alpha)$-connected and $\operatorname{dim}\left[(K-L)_{H}\right]^{\alpha} \leqslant r(H, \alpha)$ for every subgroup $H$ occurring in $M$ and any component $M_{H}^{\alpha}$ of $M_{H}$. Then there exist a compact $G$-subset $E \subset M$ and an ambient p.l. G-isotopy $h_{t}$ of $M \operatorname{rel}((M-E) \cup L)$ such that $K \subset h_{1}(U)$.

Proof. Let, for every subgroup $H$ of $G$ occurring in $M$ and any component $M_{H}^{\alpha}$ of $M_{H}, n(H, \alpha)$ be an integer such that $-3 \leqslant n(H, \alpha) \leqslant \operatorname{dim} M_{H}^{\alpha}-3$ and $n\left(g H g^{-1}, g \alpha\right)$ $=n(H, \alpha)$ for every $g \in G$, and let $n_{0}($,$) be another similar integer-valued$ function, satisfying the same properties as $n($, $)$, and such that $n_{0}(H, \alpha) \leqslant n(H, \alpha)$ $\leqslant n_{0}(H, \alpha)+1$ for all $H$ and $\alpha$. We assume inductively that the theorem holds if $r(H, \alpha) \leqslant n_{0}(H, \alpha)$ for all $H$ and $\alpha$, and we shall prove that it then holds if $r(H, \alpha) \leqslant n(H, \alpha)$ for all $H$ and $\alpha$. The theorem holds if $n_{0}(H, \alpha) \leqslant-1$ for all $H$ and $\alpha$.

If $h: M \rightarrow M$ is a $G$-homeomorphism which is $G$-isotopic to the identity we have $h\left(M_{H}^{\alpha}\right)=M_{H}^{\alpha}$ and $h\left(\left[U_{H}\right]^{\alpha}\right)=\left[h(U)_{H}\right]^{\alpha}$ for all $H$ and $\alpha$. Hence it follows, by induction, that it is enough to consider the case when

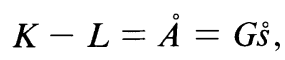

where $A$ is an equivariant simplex, in some equivariant triangulation of $L \subset K$. Then $G \dot{s}=\dot{A} \subset L \subset U$. Let $H$ be the principal isotropy subgroup of $s$ and let $M_{H}^{\alpha}$ denote

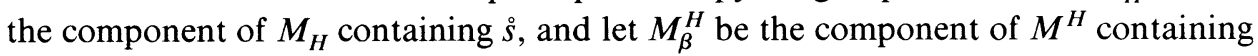
$M_{H}^{\alpha}$. Then $\stackrel{\circ}{ } \subset\left[(K-L)_{H}\right]^{\alpha}$ and we are assuming that

$$
\operatorname{dim} s=\operatorname{dim}\left[(K-L)_{H}\right]^{\alpha} \leqslant n(H, \alpha) \leqslant \operatorname{dim} M_{H}^{\alpha}-3 .
$$

If $\operatorname{dim}\left[(K-L)_{H}\right]^{\alpha} \leqslant n_{0}(H, \alpha)$, the inductive assumption applies and there is nothing to prove. Hence we can assume that $n(H, \alpha)=n_{0}(H, \alpha)+1$, and that

$$
\operatorname{dim} s=n(H, \alpha)=p .
$$

Then the pair $\left(M_{H}^{\alpha},\left[U_{H}\right]^{\alpha}\right)$ is $p$-connected. We have $\dot{s} \subset U$, and since $U$ is open there exists $\varepsilon>0$ such that

$$
s-\dot{s}_{\varepsilon} \subset U
$$

Let us denote $\sigma=s_{\varepsilon}$ and $B=G \sigma$. We now define

$$
L_{1}=L \cup(A-\stackrel{\circ}{B}) \text {. }
$$

Then $L_{1}$ is a subpolyhedron of $K$ and $K-L_{1}=\stackrel{\circ}{B}$. We have $\sigma \subset \AA \subset M_{H}^{\alpha}$ and since $\dot{\sigma} \subset U$ it follows that $\dot{\sigma} \subset\left[U_{H}\right]^{\alpha}$. Observe that the only isotropy subgroup in $\sigma$ is $H$. 
Also $B \subset \AA$ and $\dot{B} \subset U$, and the only orbit type occurring in $B$ is $(H)$. Now consider the inclusion

$$
i:(\sigma, \dot{\sigma}) \rightarrow\left(M_{H}^{\alpha},\left[U_{H}\right]^{\alpha}\right) .
$$

Since $\operatorname{dim} \sigma=p$ and $\left(M_{H}^{\alpha},\left[U_{H}\right]^{\alpha}\right)$ is $p$-connected there exists a homotopy $\xi: \sigma \times I \rightarrow$ $M_{H}$ rel $\dot{\sigma}$ from the inclusion $i$ to a map $\xi_{1}: \sigma \rightarrow\left[U_{H}\right]^{\alpha} \subset M_{H}^{\alpha}$. By p.1. approximation there exists a p.1. map

$$
\eta: \sigma \times I \rightarrow M_{\beta}^{H}
$$

such that

1. $\eta(\sigma \times I) \subset M_{H}^{\alpha}$,

2. $\eta|\sigma \times\{0\}=\xi| \sigma \times\{0\}=i$,

3. $\eta(\dot{\sigma} \times I \cup \sigma \times\{1\}) \subset\left[U_{H}\right]^{\alpha}$.

Conditions 1 and 3 are obtained by letting $\eta$ approximate $\xi$ well enough. Since $\operatorname{dim} \sigma=\operatorname{dim} s \leqslant \operatorname{dim} M_{H}^{\alpha}-3$ we have in particular $\operatorname{dim}(\sigma \times I) \leqslant \operatorname{dim} M_{H}^{\alpha}=$ $\operatorname{dim} M_{\beta}^{H}$, and hence we can approximate $\eta$ arbitrarily well by a nondegenerate p.1. map satisfying conditions 1-3 above. Thus we may as well henceforth assume that $\eta$ moreover is nondegenerate.

A triangulation $(\sigma \times I)^{*}$ of $\sigma \times I$ induces in an obvious way an equivariant triangulation $(B \times I)^{*}$ of $B \times I$. Now let $(\sigma \times I)^{*}$ be a triangulation of $\sigma \times I$, which induces a triangulation on $\sigma \times\{0\}$, and $M^{*}$ an equivariant triangulation of $M$ such that $\eta$ embeds the simplexes of $(\sigma \times I)^{*}$ and the $G$-map $\bar{\eta}:(B \times I)^{*} \rightarrow M^{*}$, defined by $\bar{\eta}(g x, t)=g \eta(x, t)$ for all $g \in G$ and $(x, t) \in \sigma \times I$, is simplicial. Since $\bar{\eta}$ is isovariant and nondegenerate in the ordinary sense it now follows by Lemma 1.7 in [10] that $\bar{\eta}$ is equivariantly nondegenerate, i.e., $\bar{\eta}$ embeds every equivariant simplex of $(B \times I)^{*}$. By Theorem 6 in Whitehead [19] there exists a subdivision $(\sigma \times I)^{* *}$ of $(\sigma \times I)^{*}$ such that $(\sigma \times I)^{* *}$ collapses simplicially to $(\dot{\sigma} \times I \cup \sigma \times\{1\})^{* *}$. Clearly $\bar{\eta}$ also embeds every equivariant simplex of $(B \times I)^{* *}$.

Let $s_{1}, \ldots, s_{k}$ be closed simplexes of $(\sigma \times I)^{* *}-(\dot{\sigma} \times I \cup \sigma \times\{1\})^{* *}$ such that, denoting

$$
\begin{aligned}
t_{j} & =(\dot{\sigma} \times I \cup \sigma \times\{1\})^{* *} \cup s_{1} \cup \cdots \cup s_{j}, \quad 1 \leqslant j \leqslant k, \quad \text { and } \\
t_{0} & =(\dot{\sigma} \times I \cup \sigma \times\{1\})^{* *},
\end{aligned}
$$

we have $t_{k}=(\sigma \times I)^{* *}$ and $t_{j} \searrow t_{j-1}$ by one elementary simplicial collapse, $j=$ $1, \ldots, k$. Thus we have

$$
t_{j-1} \cap s_{j}=v_{j} \dot{\tau}_{j}, \quad \text { where } s_{j}=v_{j} \tau_{j}, 1 \leqslant j \leqslant k .
$$

Let $t_{j}^{p}$ denote the $p$-skeleton of $t_{j}$. Observe that

$$
t_{0}=t_{0}^{p}, \text { and }(\sigma \times\{0\})^{* *} \subset t_{k}^{p} .
$$

Moreover we also have

$$
t_{j-1}^{p} \cap s_{j}=v_{j} \dot{\tau}_{j}
$$

i.e.

$$
t_{j-1}^{p} \cup s_{j} \searrow t_{j-1}^{p}
$$


by one elementary simplicial collapse, $j=1, \ldots, k$. [We have $t_{j-1}^{p} \cup s_{j} \neq t_{j}^{p}$ in the cases when $\operatorname{dim} s_{j}=p+1$.]

Let us define

$$
\begin{array}{rlrl}
S_{i} & =G s_{i}, & & i=1, \ldots, k, \\
T_{j}=G t_{j}, & & j=0, \ldots, k,
\end{array}
$$

and let $T_{j}^{p}=G t_{j}^{p}$ denote the $p$-skeleton of $T_{j}$. Then $S_{1}, \ldots, S_{k}$ are closed equivariant simplexes of $(B \times I)^{* *}-(\dot{B} \times I \cup B \times\{1\})^{* *}$. We have

$$
T_{0}=T_{0}^{p}=(\dot{B} \times I \cup B \times\{1\})^{* *}, \quad \text { and }(B \times\{0\})^{* *} \subset T_{k}^{p}
$$

and

$$
T_{j-1}^{p} \cup S_{j} \searrow T_{j-1}^{p}, \quad j=1, \ldots, k,
$$

by one elementary $G$-simplicial collapse.

Let us consider the equivariant polyhedron

$$
P=L_{1} \cup_{\dot{B} \times\{0\}}(B \times I)=K \cup_{B \times\{0\}}(B \times I) .
$$

There is an equivariant triangulation $P^{* *}$ of $P$ which is an extension of $(B \times I)^{* *}$ and which induces $K^{* *}, L_{1}^{* *}$ and $L^{* *}$ (in fact $L^{* *}=L$ ). We have the elementary $G$-simplicial collapses

$$
L_{1}^{* *} \cup T_{j-1}^{p} \cup S_{j} \searrow L_{1}^{* *} \cup T_{j-1}^{p}, \quad j=1, \ldots, k .
$$

Now consider the $G$-map

$$
\hat{\eta}: P^{* *} \rightarrow M
$$

where $\hat{\eta} \mid K^{* *}$ equals the inclusion of $K^{* *}$ into $M$ and $\hat{\eta} \mid(B \times I)^{* *}=\bar{\eta}$. Then $\hat{\eta}$ is an equivariantly nondegenerate proper p.1. $G$-map. By Proposition 3.9 in [10] there is an isovariant proper p.1. $\varepsilon$-homotopy rel $K$ from $\hat{\eta}$ to an equivariantly nondegenerate proper p.1. $G$-map $\bar{\varphi}: P^{* *} \rightarrow M$ such that $\bar{\varphi} \mid: P^{* *}-K^{* *} \rightarrow M$ is in equivariant strong general position with respect to $M$ and $K_{0} \subset M$, where $K_{0}=G \overline{\left[K_{H}\right]^{\alpha}}$. Here $\varepsilon$ denotes an arbitrary given $G$-invariant function $\varepsilon: P \rightarrow \mathbf{R}_{+}$. We have $\bar{\varphi}(\sigma \times I) \subset$ $M_{H}^{\alpha}$, and by choosing $\bar{\varphi}$ to approximate $\hat{\eta}$ well enough we get $\bar{\varphi}(\dot{\sigma} \times I \cup \sigma \times\{1\}) \subset$ $\left[U_{H}\right]^{\alpha}$. Moreover we have that if $\tau_{1}$ and $\tau_{2}$ are any two distinct simplexes of $P^{* *}-K^{* *}$ such that $\bar{\varphi}\left(\tau_{i}\right) \subset M_{\beta}^{H}, i=1,2$, then

(i) $\operatorname{dim}\left(\bar{\varphi}\left(\stackrel{\circ}{\tau}_{1}\right) \cap \bar{\varphi}\left(\stackrel{\circ}{2}_{2}\right)\right) \leqslant \operatorname{dim} \tau_{1}+\operatorname{dim} \tau_{2}-\operatorname{dim} M_{\beta}^{H}$,

(ii) $\operatorname{dim}\left(\bar{\varphi}\left(\stackrel{\circ}{\tau}_{1}\right) \cap K_{0}\right) \leqslant \operatorname{dim} \tau_{1}+\operatorname{dim}\left[\left(K_{0}\right)_{H}\right]_{\beta}-\operatorname{dim} M_{\beta}^{H}$. Here $\left[\left(K_{0}\right)_{H}\right]_{\beta}=\left(K_{0}\right)_{H} \cap M_{\beta}^{H}$.

Claim. We now claim that for each $j=0, \ldots, k$ there exist a compact $G$-subset $E_{j}$ and an ambient p.1. $G$-isotopy $h_{t}^{(j)}$ of $M \operatorname{rel}\left(\left(M-E_{j}\right) \cup L_{1}\right)$ such that

$$
\bar{\varphi}\left(T_{j}^{p}\right) \subset h_{1}^{(j)}(U)
$$

We prove this claim by induction in $j$. For $j=0$ we have $\bar{\varphi}\left(T_{0}^{\mathrm{p}}\right)=\bar{\varphi}\left(T_{0}\right) \subset U$, so we can take $h_{t}^{(0)}=\mathrm{id}$. Assume the claim holds for the value $i-1$, so that $\bar{\varphi}\left(T_{i-1}^{p}\right) \subset$ $h_{1}^{(i-1)}(U)$. We shall show that it holds for the value $i$. Consider a set of the form

$$
S\left(\bar{\varphi} \mid s_{i} \cup \tau\right),
$$


where $\tau$ is any ordinary simplex of $L_{1}^{* *} \cup T_{i-1}^{p}$. (Here $S\left(\varphi \mid s_{i} \cup \tau\right.$ ) denotes the singular set of the map $\varphi \mid s_{i} \cup \tau$, see e.g. Rushing [13, p. 28].) Since $\bar{\varphi}$ is nondegenerate it is dimension preserving. Hence it follows from (i) that if $\tau$ is a simplex of $T_{i-1}^{p}-L_{1}^{* *}$ we have

$$
\operatorname{dim} S\left(\bar{\varphi} \mid s_{i} \cup \tau\right) \leqslant \operatorname{dim} s_{i}+\operatorname{dim} \tau-\operatorname{dim} M_{\beta}^{H} \leqslant p+1+p-\operatorname{dim} M_{\beta}^{H} \leqslant p-2,
$$

since $p \leqslant \operatorname{dim} M_{H}^{\alpha}-3=\operatorname{dim} M_{\beta}^{H}-3$. If $\tau$ is a simplex of $L_{1}^{* *}$ we have $S\left(\bar{\varphi} \mid s_{i} \cup \tau\right)$ $=\varnothing$ unless $\tau=\bar{\varphi}(\tau) \subset M_{H}^{\alpha}$, i.e., $\tau \subset\left[K_{H}\right]^{\alpha} \subset K_{0}$. In this case we have by (ii)

$$
\begin{aligned}
\operatorname{dim} S\left(\bar{\varphi} \mid s_{i} \cup \tau\right) & \leqslant \operatorname{dim}\left(\bar{\varphi}\left(s_{i}\right) \cap K_{0}\right) \\
& \leqslant \operatorname{dim} s_{i}+\operatorname{dim}\left[\left(K_{0}\right)_{H}\right]_{\beta}-\operatorname{dim} M_{\beta}^{H} \leqslant p-2,
\end{aligned}
$$

since $\operatorname{dim} K_{0}=\operatorname{dim}\left[K_{H}\right]^{\alpha} \leqslant \operatorname{dim} M_{H}^{\alpha}-3=\operatorname{dim} M_{\beta}^{H}-3$.

Let us denote

$$
\Sigma_{i}^{\prime}=\left[\bigcup_{i} S\left(\bar{\varphi} \mid s_{i} \cup \tau\right)\right] \cap s_{i}
$$

where the union is over all ordinary simplexes $\tau$ of $L_{1}^{* *} \cup T_{i-1}^{p}$. Then $\Sigma_{i}^{\prime}$ is a subpolyhedron of $s_{i}$ and $\operatorname{dim} \Sigma_{i}^{\prime} \leqslant p-2$. Since $t_{i-1}^{p} \cup s_{i}$ collapses (by an elementary simplicial collapse) to $t_{i-1}^{p}$ there exists (see Lemma 1.6.3 in Rushing [13]) a subpolyhedron $\Lambda_{i}^{\prime} \subset s_{i}$ with $\Sigma_{i}^{\prime} \subset \Lambda_{i}^{\prime}$ such that $t_{i-1}^{p} \cup s_{i}$ collapses to $t_{i-1}^{p} \cup \Lambda_{i}^{\prime}$ and $\operatorname{dim} \Lambda_{i}^{\prime} \leqslant p-1$. Hence in some subdivision $\left(t_{i-1}^{p} \cup s_{i}\right)^{\oplus}$ of $t_{i-1}^{p} \cup s_{i}$, such that $\left(t_{i-1}^{p} \cup \Lambda_{i}^{\prime}\right)^{\oplus}$ is a subcomplex, we have that $\left(t_{i-1}^{p} \cup s_{i}\right)^{\oplus}$ collapses simplicially to $\left(t_{i-1}^{p} \cup \Lambda_{i}^{\prime}\right)^{\oplus}$. Let $\Lambda_{i}=G \Lambda_{i}^{\prime} \subset S_{i}$ and $\Sigma_{i}=G \Sigma_{i}^{\prime} \subset \Lambda_{i}$. Then $\left(T_{i-1}^{p} \cup S_{i}\right)^{\oplus}$ collapses $G$-simplicially to $\left(T_{i-1}^{p} \cup \Lambda_{i}\right)^{\oplus}$. The equivariant subdivision $\left(T_{i-1}^{p} \cup S_{i}\right)^{\oplus}$ can be extended to an equivariant subdivision $\left(L_{1} \cup T_{i-1}^{p} \cup S_{i}\right)^{\oplus}$ of $L_{1}^{* *} \cup T_{i-1}^{p} \cup S_{i}$. Then $\left(L_{1} \cup T_{i-1}^{p} \cup S_{i}\right)^{\oplus}$ collapses $G$-simplicially to $\left(L_{1} \cup T_{i-1}^{p} \cup \Lambda_{i}\right)^{\oplus}$. Let

$$
\left(L_{1} \cup T_{i-1}^{p} \cup S_{i}\right)^{\oplus}=R_{d} \searrow R_{d-1} \searrow \cdots \searrow R_{0}=\left(L_{1} \cup T_{i-1}^{p} \cup \Lambda_{i}\right)^{\oplus}
$$

be a sequence of elementary $G$-simplicial collapses. Let us now show that there is an elementary equivariant collapse from $\bar{\varphi}\left(R_{e}\right)$ to $\bar{\varphi}\left(R_{e-1}\right)$ for $e=1, \ldots, d$. Let $R_{e}-$ $R_{e-1}$ consist of the two equivariant simplexes $G w$ and $G w_{1}$ with $w_{1}$ a face of $w$ and $w=a w_{1}$, where $a$ is a vertex of $w$, and where we have chosen the ordinary simplex $w$ such that $w \subset s_{i}$. (Thus both $w_{1}$ and $w$ have principal isotropy subgroup equal to $H$.) We have $w \cap R_{e-1}=a \dot{w}_{1}$, and $\bar{\varphi} \mid: G w \rightarrow M$ is a p.1. $G$-imbedding. We claim that $p \mid: \bar{\varphi}(w) \rightarrow M / G$ is injective. For if $x, y \in w$ and $p(\bar{\varphi}(x))=p(\bar{\varphi}(y))$ we have $\bar{\varphi}(x)=g \bar{\varphi}(y)$ for some $g \in G$ and hence $\bar{\varphi}(x)=\bar{\varphi}(g y)$ implies $x=g y$. Since $y \in w$ and $g y=x \in w$ it follows that $g \in H$ and $x=y$. Next we claim that $x \in w-R_{e-1}$ implies that $\bar{\varphi}(x) \notin \bar{\varphi}\left(R_{e-1}\right)$. For if $z \in R_{e-1}$ and $\bar{\varphi}(x)=\bar{\varphi}(z)$ we cannot have $z \in L_{1} \cup T_{i-1}^{p}$ since $x \in s_{i}-\Lambda_{i}^{\prime} \subset s_{i}-\Sigma_{i}^{\prime}$, and hence $z \in S_{i}$ which since $\bar{\varphi} \mid S_{i}$ is a $G$-imbedding implies that $x=z$, a contradiction. It now follows that $\bar{\varphi}(w) \cap$ $\bar{\varphi}\left(R_{e-1}\right)=\bar{\varphi}\left(w \cap R_{e-1}\right)=\bar{\varphi}\left(a \dot{w}_{1}\right)$. Thus $\bar{\varphi}(w)$ is a $q$-ball $(q=\operatorname{dim} w)$ in $\bar{\varphi}\left(R_{e}\right)$ which injects into the orbit space such that $\bar{\varphi}(w) \cap \bar{\varphi}\left(R_{e-1}\right)=\bar{\varphi}\left(a \dot{w}_{1}\right)$ is a $(q-1)$ ball in $\partial(\bar{\varphi}(w))$ and $\bar{\varphi}\left(R_{e}\right)=\bar{\varphi}\left(R_{e-1}\right) \cup G \bar{\varphi}(w)$. This shows that there is an elementary equivariant collapse from $\bar{\varphi}\left(R_{e}\right)$ to $\bar{\varphi}\left(R_{e-1}\right)$.

Thus $\bar{\varphi}\left(L_{1} \cup T_{i-1}^{p} \cup S_{i}\right)$ collapses equivariantly to $\bar{\varphi}\left(L_{1} \cup T_{i-1}^{p} \cup \Lambda_{i}\right)$. Since $\bar{\varphi}(\sigma \times I) \subset M_{H}$ it follows that $\left[\left(\bar{\varphi}\left(L_{1} \cup T_{i-1}^{p} \cup \Lambda_{i}\right)-\bar{\varphi}\left(L_{1} \cup T_{i-1}^{p}\right)\right)_{H^{\prime}}\right]^{\gamma} \neq \varnothing$ only 
if $H^{\prime}=g H g^{-1}$ and $\gamma=g \alpha$, for some $g \in G$. Since also $\operatorname{dim}\left(\bar{\varphi}\left(L_{1} \cup T_{i-1}^{p} \cup \Lambda_{i}\right)-\right.$ $\left.\bar{\varphi}\left(L_{1} \cup T_{i-1}^{p}\right)\right) \leqslant p-1$, we have by our main inductive assumption that the theorem holds in this case, i.e., there exist a compact $G$-subset $\tilde{E}_{i}$ and an ambient p.1. $G$-isotopy $\tilde{h}^{(i)} \operatorname{rel}\left(\left(M-\tilde{E}_{i}\right) \cup \bar{\varphi}\left(L_{1} \cup T_{i-1}^{p}\right)\right)$ such that

$$
\tilde{h}_{1}^{(i)}\left(h_{1}^{(i-1)}(U)\right) \supset \bar{\varphi}\left(L_{1} \cup T_{i-1}^{p} \cup \Lambda_{i}\right) \text {. }
$$

Since $\bar{\varphi}\left(L_{1} \cup T_{i-1}^{p} \cup S_{i}\right)$ collapses equivariantly to $\bar{\varphi}\left(L_{1} \cup T_{i-1}^{p} \cup \Lambda_{i}\right)$ it now follows by Proposition 2.3 that there exist a compact $G$-subset $\hat{E}_{1}$ and an ambient p.1. $G$-isotopy $\hat{h}^{(i)} \operatorname{rel}\left(\left(M-\hat{E}_{i}\right) \cup \bar{\varphi}\left(L_{1} \cup T_{i-1}^{p} \cup \Lambda_{i}\right)\right)$ such that

$$
\hat{h}_{1}^{(i)}\left(\tilde{h}_{1}^{(i)} h_{1}^{(i-1)}(U)\right) \supset \bar{\varphi}\left(L_{1} \cup T_{i-1}^{p} \cup S_{i}\right) \text {. }
$$

Now let $E_{i}=\hat{E}_{i} \cup \tilde{E}_{i} \cup E_{i-1}$ and $h^{(i)}=\hat{h}^{(i)} \circ \tilde{h}^{(i)} \circ h^{(i-1)}$, then $h^{(i)}$ is an ambient p.1. $G$-isotopy such that $h_{1}^{(i)}(U) \supset \bar{\varphi}\left(L_{1} \cup T_{i-1}^{p} \cup S_{i}\right) \supset \bar{\varphi}\left(L_{1} \cup T_{i}^{p}\right)$. This completes the inductive proof of our "claim". Thus $h=h^{(k)}$ is an ambient p.1. $G$-isotopy $\operatorname{rel}\left(\left(M-E_{k}\right) \cup L\right)$ such that

$$
h_{1}(U) \supset \bar{\varphi}\left(L_{1} \cup T_{k}^{p}\right) \supset \bar{\varphi}\left(L_{1} \cup(B \times\{0\})\right)=\bar{\varphi}(K)=K .
$$

We will also use the following version of the equivariant engulfing theorem. (This version of the equivariant engulfing theorem corresponds to the version of the ordinary engulfing theorem given in Theorem 6.1 in Stallings [17].)

THEOREM 2.5. Let $M$ be an equivariant p.l. manifold, without boundary. Let $U$ be an open $G$-subset of $M$ and $C$ a closed $G$-subset of $M$ such that $C \subset U$. Let $K$ be an equivariant subpolyhedron of $M$ such that $K-U$ is compact. Assume that

$$
\left(\left[(M-C)_{H}\right]^{\alpha},\left[(U-C)_{H}\right]^{\alpha}\right)
$$

is $r(H, \alpha)$-connected and $\operatorname{dim}\left[(K-C)_{H}\right]^{\alpha} \leqslant r(H, \alpha) \leqslant \operatorname{dim} M_{H}^{\alpha}-3$ for every subgroup $H$ and component $M_{H}^{\alpha}$ of $M_{H}$ for which $\left[(K-C)_{H}\right]^{\alpha} \neq \varnothing$. Then there exist a compact $G$-subset $E \subset M-C$ and an ambient p.l. G-isotopy $h_{t}$ of $M \operatorname{rel}(M-E)$ such that $K \subset h_{1}(U)$.

Proof. Denote $M_{1}=M-C, U_{1}=U-C$ and $K_{1}=K-C$. Then $K_{1}$ is an equivariant subpolyhedron of the equivariant p.1. manifold $M_{1}$. Let $K_{1}$ be given some equivariant triangulation and let $L_{1}$ denote the equivariant subcomplex of $K_{1}$ consisting of all closed simplexes of $K_{1}$ that are contained in $U_{1}$. Then $L_{1} \subset U_{1}$ and $\overline{K_{1}-L_{1}}=N\left(K_{1}-U_{1} ; K_{1}\right)$ is compact since $K_{1}-U_{1}=K-U$ is compact. The proof is now completed by applying Theorem 2.4 to the situation $\left(M_{1}, U_{1}, L_{1}, K_{1}\right)$. (Observe the following: Let $H$ be a subgroup of $G$ and $\left(M_{1}\right)_{H}^{\gamma}$ a component of $\left(M_{1}\right)_{H}$ such that $\left[\left(K_{1}-L_{1}\right)_{H}\right]^{\gamma} \neq \varnothing$. Let $M_{H}^{\alpha}$ be the component of $M_{H}$ containing $\left(M_{1}\right)_{H}^{\gamma}$. Then $\varnothing \neq\left[\left(K_{1}\right)_{H}\right]^{\gamma} \subset\left[\left(K_{1}\right)_{H}\right]^{\alpha}$ and hence, by the assumption in 2.5 and the fact that $\operatorname{dim} M_{H}^{\alpha}=\operatorname{dim}\left(M_{1}\right)_{H}^{\gamma}$, we have $\operatorname{dim}\left[\left(K_{1}\right)_{H}\right]^{\gamma} \leqslant \operatorname{dim}\left[\left(K_{1}\right)_{H}\right]^{\alpha} \leqslant \operatorname{dim} M_{H}^{\alpha}-3=$ $\operatorname{dim}\left(M_{1}\right)_{H}^{\gamma}-3$.)

3. Recognition of linear actions on euclidean space. In this section we prove Proposition 3.2 which gives necessary and sufficient conditions for an equivariant p.1. manifold $M$ to be $G$-homeomorphic to some euclidean representation space. Proposition 3.2 below is in fact given in a more general form than we would need. In 
the situation that we use Proposition 3.2 we are already given that the underlying manifold is euclidean space and that all fixed-point sets are homeomorphic to euclidean space. There is also a version of the result in Proposition 3.2 that gives necessary and sufficient conditions for $M$ to be p.1. $G$-homeomorphic to some euclidean representation space, but we have no use for this p.1. version in this paper, and hence we omit it.

Definition 3.1. Let $M$ be an equivariant p.1. manifold and $\mathscr{F}$ a family of subgroups of $G$, occurring in $M$, which is closed under conjugation. We say that $M$ is $\mathscr{F}$-equivariantly unraveled at infinity if given any compact subset $A$ of $M$ there exists a compact $G$-subset $B$ of $M$, with $A \subset B$, such that $\left(M_{H},(M-B)_{H}\right)$ is 2-connected for every $H \in \mathcal{F}$ for which $M^{H}$ is noncompact. In case $\mathscr{F}$ is the family of all subgroups occurring in $M$ we simply use the expression "equivariantly unraveled at infinity" instead of "F్F-equivariantly unraveled at infinity".

Proposition 3.2. Let $M$ be an equivariant p.l. manifold, without boundary, such that $\operatorname{dim} M^{H} \geqslant 5$ for every subgroup $H \neq G$ occurring in $M$. Then $M$ is $G$-homeomorphic to some orthogonal representation space $\mathbf{R}^{m}(\rho)$ if and only if the following three conditions are satisfied.

I. The case $0 \leqslant \operatorname{dim} M^{G} \leqslant 4$.

1. $M^{G}$ is homeomorphic to $\mathbf{R}^{k}$, where $k=\operatorname{dim} M^{G}$.

2. $M$ is $\mathscr{F}^{\prime}$-equivariantly unraveled at infinity, where $\mathscr{F}^{\prime}=\mathscr{F}-\{G\}$ and $\mathscr{F}$ denotes the family of all subgroups occurring in $M$.

3. There is a closed G-invariant neighbourhood $D$ of $M^{G}$ in $M$, with $D$ G-homeomorphic to some $\mathbf{R}^{k} \times B^{m-k}\left(\rho^{\prime}\right)$, such that $\left(M_{H}, D_{H}\right)$ is q-connected for all $q$ and every $H \in \mathscr{F}^{\prime}$.

II. The case $\operatorname{dim} M^{G} \geqslant 5$.

1. $M^{G}$ is contractible.

2. $M$ is equivariantly unraveled at infinity.

3. There exists an open $G$-subset $U$ of $M G$-homeomorphic to some $\mathbf{R}^{m}(\rho)$ such that $\left(M_{H}, U_{H}\right)$ is q-connected for all $q$ and every $H \in \mathcal{F}^{\prime}$. (Observe that in this case $\left(M_{G}, U_{G}\right)=\left(M^{G}, U^{G}\right)$ is also automatically $q$-connected for all $q$. $)$

Proof. The "only if" part is clear.

In order to prove the "if" part we will first prove that given any compact subset $A$ of $M$ there exists an equivariant ball $E$ in $M$ such that $A \subset E$.

I. The case $0 \leqslant \operatorname{dim} M^{G} \leqslant 4$. Let $k=\operatorname{dim} M^{G}$ and let $\alpha: \mathbf{R}^{k} \times B^{m-k}\left(\rho^{\prime}\right) \rightarrow D$ be a $G$-homeomorphism. Then we have $M^{G}=D^{G}=\alpha\left(\mathbf{R}^{k} \times\{0\}\right)$. Let us define

$$
\begin{aligned}
C & =\alpha\left(\mathbf{R}^{k} \times B_{1 / 2}^{m-k}\left(\rho^{\prime}\right)\right), \quad \text { and } \\
W & =M-\alpha\left(\mathbf{R}^{k} \times B_{1 / 3}^{m-k}\left(\rho^{\prime}\right)\right),
\end{aligned}
$$

where $B_{r}^{m-k}\left(\rho^{\prime}\right)=\left\{x \in \mathbf{R}_{r}^{m-k}\left(\rho^{\prime}\right) \mid\|x\| \leqslant r\right\}$. Then $W$ is open in $M$ and Int $C$ $=\alpha\left(\mathbf{R}^{k} \times \ddot{B}_{1 / 2}^{m-k}\left(\rho^{\prime}\right)\right)$. Observe that $M=\operatorname{Int} C \cup W$. Let us denote $U=\operatorname{Int} D=$ $\alpha\left(\mathbf{R}^{k} \times \dot{B}^{m-k}\left(\rho^{\prime}\right)\right)$. Then $M^{G} \subset C \subset U$.

Now let $A$ be an arbitrary compact subset of $M$. Since $M$ is $\mathscr{F}^{\prime}$-equivariantly unraveled at infinity there exists a compact $G$-subset $B$, where $A \subset B$, such that 
$\left(M_{H},(M-B)_{H}\right)$ is 2-connected for every subgroup $H \neq G$ occurring in $M$ for which $M^{H}$ is noncompact. If $1 \leqslant \operatorname{dim} M^{G} \leqslant 4$, then $M^{G}$ is noncompact, and hence in this case $M^{H}$ is noncompact for every subgroup $H$. If $\operatorname{dim} M^{G}=0$ then $M^{G}=\{a\}$, a point, and we can assume that $B$ is chosen such that $A \cup\{a\} \subset B$. Then we have $(M-B)^{G}=\varnothing$. We claim that $M^{H}$ is noncompact for every subgroup $H \neq G$ occurring in $M$. Let $H_{1}$ be a subgroup of $G$ which occurs in $M$ and which is maximal in the set of all subgroups different from $G$ occurring in $M$. Then $M^{H_{1}}=M_{H_{1}} \cup M^{G}$ $=M_{H_{1}} \cup\{a\}$ and $D^{H_{1}}=D_{H_{1}} \cup D^{G}=D_{H_{1}} \cup\{a\}$. Let us denote $k_{1}=\operatorname{dim} M^{H_{1}} \geqslant 5$. If $M^{H_{1}}$ were compact we would have $H_{k_{1}}\left(M^{H_{1}} ; Z_{2}\right) \cong Z_{2}$. Since $\left(M_{H_{1}}, D_{H_{1}}\right)$ is $q$-connected for all, it follows by excision that $H_{k_{1}}\left(M^{H_{1}}, D^{H_{1}} ; Z_{2}\right) \cong$ $H_{k_{1}}\left(M_{H_{1}}, D_{H_{1}} ; Z_{2}\right)=0$. Therefore by the exact homology sequence of the pair $\left(M^{H_{1}}, D^{H_{1}}\right)$, we get $H_{k_{1}}\left(M^{H_{1}} ; Z_{2}\right) \cong H_{k_{1}}\left(M^{H_{1}}, D^{H_{1}} ; Z_{2}\right)=0$. Thus $M^{H_{1}}$ is noncompact. It now follows that $M^{H}$ is noncompact for every subgroup $H \neq G$ occurring in $M$.

Let $T$ be an equivariant triangulation of $M$ which is subordinate to the open cover \{Int $C, W$ \}. Let $L$ be the subcomplex of $T$ which consists of all closed simplexes of $T$ that are contained in $W$. Since $W^{G}=\varnothing$ we have $L^{G}=\varnothing$. Let us denote

$$
V=M-B \text {. }
$$

Then $M-V=B$ is compact. Let $L^{2}$ be the 2-skeleton of $L$. Thus $L^{2}-V$ is compact and $\left(M_{H}, V_{H}\right)$ is 2-connected for every $H$ which occurs in $L$. Hence there exist, by Theorem 2.4, a compact $G$-subset $E_{1}^{\prime}$ of $M$ and a $G$-homeomorphism $v$ of $M$ such that $L^{2} \subset v(V)$ and $v \mid M-E_{1}^{\prime}=$ id. Let $E_{1}=E_{1}^{\prime} \cup B$. Then we have $v \mid M-$ $E_{1}=$ id and $M-E_{1} \subset V$. We now define $P$ to be the union of all closed simplexes of $T$ that are contained in $M-E_{1}$, and

$$
K=L^{2} \cup P .
$$

Then, since $L^{2} \subset v(V)$ and $P \subset M-E_{1}=v\left(M-E_{1}\right) \subset v(V)$, it follows that

$$
K \subset v(V) \text {. }
$$

Now define

$$
J=T \div K
$$

i.e., $J$ is the maximal subcomplex of the first barycentric subdivision $T^{\prime}$ of $T$ which does not intersect $K$. We have

$$
J \subset T-K \subset T-P \subset N\left(E_{1} ; T\right),
$$

and hence $J$ is compact. Next observe that

$$
J-C \subset\left(T \div L^{2}\right)-C .
$$

We claim that for every subgroup $H$ of $G$ and any component $M_{H}^{\alpha}$ of $M_{H}$ we have

$$
\operatorname{dim}\left[(J-C)_{H}\right]^{\alpha} \leqslant \operatorname{dim} M_{H}^{\alpha}-3 .
$$

Because of $(*)$ it is enough to prove $(* *)$ with $\left(T \div L^{2}\right)-C$ in place of $J-C$. Let $s^{\prime}$ be a simplex of $T \div L^{2}$ with principal isotropy subgroup equal to $H$ and such that $s^{\prime} \subset M_{H}^{\alpha}$ and $s^{\prime} \nsubseteq C$. Then $s^{\prime}$ is a simplex of $T^{\prime}$ such that $s^{\prime} \cap L^{2}=\varnothing$. Let $s$ be the carrier of $s^{\prime}$ in $T$, i.e. $s$ is the smallest simplex of $T$ which contains $s^{\prime}$. Then we also 
have $s \nsubseteq C$ and hence in particular $s \nsubseteq$ Int $C$. Thus $s \subset W$, since $T$ is subordinate to the open cover $\{$ Int $C, W\}$. Since the principal isotropy subgroup of $s$ also equals $H$ we have $s \subset M_{H}$. Since $s \subset W$ we have $s \subset L$, and hence the 2-skeleton $s^{2}$ of $s$ lies in $L^{2}$. Then $s^{\prime} \subset s$ and $s^{\prime} \cap L^{2}=\varnothing$ imply that

$$
\operatorname{dim} s^{\prime} \leqslant \operatorname{dim} s-3 \leqslant \operatorname{dim} M_{H}^{\alpha}-3 .
$$

The pair $(M-C, U-C)$ is $G$-homeomorphic to $\left(M-M^{G}, U-M^{G}\right)$. Thus $\left((M-C)_{H},(U-C)_{H}\right)$ is homeomorphic to $\left(M_{H}, U_{H}\right)$ for every $H \neq G$. Hence $\left((M-C)_{H},(U-C)_{H}\right)$ is $q$-connected for all $q$ and every subgroup $H$ occurring in $J-C$. It now follows, by Theorem 2.5 , that there is a $G$-homeomorphism $u$ of $M$ such that $J \subset u(U)$.

Since $K \subset v(V)$ and $J \subset u(U)$ and $J=T \div K$ there exists a $G$-homeomorphism $w:\left|T^{\prime}\right| \rightarrow\left|T^{\prime}\right|$ such that

$$
w(u(U)) \cup v(V)=\left|T^{\prime}\right|=M .
$$

Then

$$
h=v^{-1} \circ w \circ u: M \rightarrow M
$$

is a $G$-homeomorphism such that $h(U) \cup V=M$, and hence

$$
A \subset B \subset h(U) \text {. }
$$

Since $A$ is compact and $h(U) \subset h(D)=(h \circ \alpha)\left(\mathbf{R}^{k} \times B^{m-k}\left(\rho^{\prime}\right)\right)$ there exists $r>0$ such that

$$
A \subset(h \circ \alpha)\left(B_{r}^{k} \times B^{m-k}\left(\rho^{\prime}\right)\right)=E,
$$

where $B_{r}^{k}=\left\{x \in \mathbf{R}^{k} \mid\|x\| \leqslant r\right\}$. Then $E$ is $G$-homeomorphic with $B^{m}(\rho)$, where

$$
\rho=\underbrace{\mathrm{id} \oplus \cdots \oplus \mathrm{id}}_{k} \oplus \rho^{\prime},
$$

and we have thus proved that $A$ is contained in an equivariant ball $E$ in $M$.

In the case when $\operatorname{dim} M^{G} \geqslant 5$ the proof of the fact that any compact subset $A$ of $M$ is contained in an equivariant ball $E$ is completely similar to the above proof and in fact simpler. The sets $C$ and $W$ are not needed at all and $L$ is chosen to be $T$.

Thus it follows that in both cases I and II we have that $M=\cup_{i=1}^{\infty} E_{i}$, where $E_{1} \subset \stackrel{\circ}{E}_{2} \subset E_{2} \subset \stackrel{\circ}{E}_{3} \subset \ldots$ is an increasing sequence of equivariant $m$-balls and each $E_{i}$ is $G$-homeomorphic to $B^{m}(\rho), i=1,2,3, \ldots$ Hence Proposition 3.4 below implies that $M$ is $G$-homeomorphic to $\mathbf{R}^{m}(\rho)$.

The proof of Proposition 3.4 below is completely analogous to the proof of the "monotone union of open $n$-cells theorem" by Morton Brown [3]. If $S$ and $S^{\prime}$ are two embedded nonintersecting $(m-1)$-spheres in an $m$-ball we denote by $\left[S, S^{\prime}\right]$ the closure of the region between $S$ and $S^{\prime}$. The crucial lemma is the following.

LEMmA 3.3. Let $j: B^{n}(\rho) \rightarrow B^{n}(\rho)$ be a G-embedding such that $j\left(B^{n}(\rho)\right) \subset B^{n}(\rho)$, and let $A \subset B^{n}(\rho)$ be compact. Then there exists a G-homeomorphism $h: B^{n}(\rho) \rightarrow$ $B^{n}(\rho)$ such that $j\left(B^{n}(\rho)\right) \cup A \subset h j\left(B_{1 / 2}^{n}(\rho)\right)$ and $\left[j\left(S_{1 / 2}^{n-1}(\rho)\right), h j\left(S_{1 / 2}^{n-1}(\rho)\right)\right]$ is $G$ homeomorphic to $S^{n-1}(\rho) \times[0,1]$. 
Proof. We first prove the lemma in the case that $j(0)=0$. Let $\varepsilon>0$ be such that $B_{\varepsilon}^{n}(\rho) \subset j\left(B_{1 / 2}^{n}(\rho)\right)$ and $j\left(B^{n}(\rho)\right) \cup A \subset B_{1-\varepsilon}^{n}(\rho)$. Let $h: B^{n}(\rho) \rightarrow B^{n}(\rho)$ be a $G$ homeomorphism such that $h \mid B_{\varepsilon / 2}^{n}(\rho)=\mathrm{id}, h\left(B_{\varepsilon}^{n}(\rho)\right)=B_{1-\varepsilon}^{n}(\rho)$ and $h \mid S^{n-1}(\rho)=$ id. Then $j\left(B^{n}(\rho)\right) \cup A \subset B_{1-\varepsilon}^{n}(\rho)=h\left(B_{\varepsilon}^{n}(\rho)\right) \subset h j\left(B_{1 / 2}^{n}(\rho)\right)$. It remains to prove that $\left[j\left(S_{1 / 2}^{n-1}(\rho)\right), h j\left(S_{1 / 2}^{n-1}(\rho)\right)\right]$ is $G$-homeomorphic to $S^{n-1}(\rho) \times[0,1]$.

Choose a $G$-homeomorphism $f^{\prime}: j\left(B^{n}(\rho)\right) \rightarrow j\left(B^{n}(\rho)\right)$ such that $f^{\prime} \mid j\left(S^{n-1}(\rho)\right)=$ id, $f^{\prime}\left(j\left(S_{3 / 4}^{n-1}(\rho)\right)\right)=j\left(S_{1 / 2}^{n-1}(\rho)\right)$ and $f^{\prime}\left(j\left(B_{1 / 2}^{n}(\rho)\right)\right) \subset B_{\varepsilon / 2}^{n}(\rho)$, and extend $f^{\prime}$ by the identity map to a $G$-homeomorphism $f: B^{n}(\rho) \rightarrow B^{n}(\rho)$. Then we have the following sequence of $G$-homeomorphisms

$$
\begin{aligned}
S^{n-1}(\rho) \times[0,1] & \rightarrow\left[S_{1 / 2}^{n-1}(\rho), S_{3 / 4}^{n-1}(\rho)\right] \underset{\cong}{\stackrel{j}{\cong}}\left[j\left(S_{1 / 2}^{n-1}(\rho)\right), j\left(S_{3 / 4}^{n-1}(\rho)\right)\right] \\
& \stackrel{f}{\cong}\left[f j\left(S_{1 / 2}^{n-1}(\rho)\right), j\left(S_{1 / 2}^{n-1}(\rho)\right)\right] \stackrel{h}{\cong}\left[f j\left(S_{1 / 2}^{n-1}(\rho)\right), h j\left(S_{1 / 2}^{n-1}(\rho)\right)\right] \\
& \stackrel{f^{-1}}{\cong}\left[j\left(S_{1 / 2}^{n-1}(\rho)\right), h j\left(S_{1 / 2}^{n-1}(\rho)\right)\right] . \\
& \cong
\end{aligned}
$$

Now consider the case when $j(0)=b \neq 0$. Then $G_{b}=G$ and the line $\mathbf{R} b$ is pointwise fixed under the action of $G$, i.e. $\mathbf{R} b \subset\left(\mathbf{R}^{n}(\rho)\right)^{G}$. Then we can write $\mathbf{R}^{n}(\rho)=\mathbf{R}^{n-1}\left(\rho^{\prime}\right) \oplus \mathbf{R} b$ and it follows immediately that there exists a $G$-homeomorphism $k: B^{n}(\rho) \rightarrow B^{n}(\rho)$ such that $k(b)=0$. Then applying the above considered case to the $G$-imbedding $k \circ j$ and the compact set $k(A)$ we find a $G$-homeomorphism $h^{\prime}: B^{n}(\rho) \rightarrow B^{n}(\rho)$ such that $k j\left(B^{n}(\rho)\right) \cup k(A) \subset h^{\prime} k j\left(B_{1 / 2}^{n}(\rho)\right)$ and $\left[k j\left(S_{1 / 2}^{n-1}(\rho)\right), h^{\prime} k j\left(S_{1 / 2}^{n-1}(\rho)\right)\right]$ is $G$-homeomorphic to $S^{n-1}(\rho) \times[0,1]$. Thus the $G$ homeomorphism $h=k^{-1} \circ h^{\prime} \circ k$ is of the required form.

Proposition 3.4. Let $M$ be a topological m-manifold with an action of $G$. Assume that $M=\cup_{i=1}^{\infty} E_{i}$, where $E_{1} \subset \dot{E}_{2} \subset E_{2} \subset \dot{E}_{3} \subset \cdots$ and there exist G-homeomorphisms $f_{i}: B^{m}(\rho) \rightarrow E_{i}$ and $\stackrel{\circ}{E}_{i}=f_{i}\left(\stackrel{\circ}{B}^{m}(\rho)\right)$, for all $i$. Then $M$ is G-homeomorphic to $\mathbf{R}^{m}(\rho)$.

Proof. Let us denote $E_{1}=E, f_{1}\left(B_{1 / 2}^{m}(\rho)\right)=E(1 / 2) \subset E$ and $f_{1}\left(S_{1 / 2}^{m}(\rho)\right)=$ $\partial E(1 / 2)$, and let $\cong$ stand for "is $G$-homeomorphic to". Consider the $G$-embedding $E=E_{1} \hookrightarrow E_{2}$. Then Lemma 3.3 implies that there exists a $G$-homeomorphism $k_{2}: E_{2} \rightarrow E_{2}$ such that $E \subset k_{2}(E(1 / 2))$ and $\left[\partial E(1 / 2), k_{2} \partial E(1 / 2)\right] \cong S^{m-1}(\rho) \times$ $[0,1]$. Then consider the $G$-embedding $k_{2} \mid: E \rightarrow E_{3}, x \mapsto k_{2}(x)$. By Lemma 3.3 there exists a $G$-homeomorphism $k_{3}: E_{3} \rightarrow E_{3}$ such that $E_{2} \subset k_{3} k_{2}(E(1 / 2))$ and

$$
\left[k_{2} \partial E(1 / 2), k_{3} k_{2} \partial E(1 / 2)\right] \cong S^{m-1}(\rho) \times[0,1] .
$$

Next consider the $G$-embedding $k_{3} k_{2} \mid: E \rightarrow E_{4}$, and so on. In this way we see that there exist $G$-homeomorphisms $k_{i}$ of $E_{i}$, where $k_{1}=\mathrm{id}$, such that denoting

$$
s_{i}=k_{i} \circ \cdots \circ k_{2} \circ k_{1}: E \rightarrow M
$$

we have that

$$
\left\{\begin{array}{l}
E_{i-1} \subset s_{i}(E(1 / 2)), \\
{\left[s_{i-1} \partial E(1 / 2), s_{i} \partial E(1 / 2)\right] \cong S^{m-1}(\rho) \times[0,1],}
\end{array} \quad i=2,3, \ldots\right.
$$


Thus $M=\cup_{i=1}^{\infty} s_{i}(E(1 / 2))$. It now follows that there is a continuous bijective $G$-map $h: \mathbf{R}^{m}(\rho) \rightarrow M$, and hence, by invariance of domain, $h$ is a $G$-homeomorphism.

4. The main lemmas. In this section we prove the main lemmas that allow us to handle codimension 2 fixed-point situations and the interference of codimension 1 and codimension 2 fixed-point situations. We will often encounter nonconnected manifolds. Any notion we use for a nonconnected manifold $W$ is defined in the obvious way, i.e., by the requirement that it holds for every component $V$ of $W$. For example, we say that a nonconnected manifold $W$ is orientable if every component $V$ of $W$ is orientable, and $p: \tilde{W} \rightarrow W$ is a universal covering of $W$ if $p \mid: p^{-1}(V) \rightarrow V$ is a universal covering of $V$ for every component $V$ of $W$.

LEMMA 4.1. Let $M$ be an orientable manifold and $W$ an open subset of $M$. Let $\Re$ be a finite family of orientable closed submanifolds of $M$ which is closed under intersection. Assume that $i_{*}: H_{q}(W) \rightarrow H_{q}(M)$ and $i_{*}^{\prime}: H_{q}(W \cap N) \rightarrow H_{q}(N)$ are isomorphisms for all $q \geqslant 0$, and every $N \in \mathcal{N}$, where $i$ and $i^{\prime}$ denote inclusions. Then

$$
j_{*}: H_{q}\left(W-\bigcup_{i=1}^{r}\left(W \cap N_{i}\right)\right) \rightarrow H_{q}\left(M-\bigcup_{i=1}^{r} N_{i}\right) \text {, }
$$

where $j$ denotes the inclusion, is an isomorphism for all $q \geqslant 0$ and any $N_{1}, \ldots, N_{r} \in \Re$.

Proof. Using homology Mayer-Vietoris sequences and the 5-lemma we see that it is enough to consider the case $r=1$. Let $N \in \mathfrak{N}$. Once again using homology Mayer-Vietoris sequences and the 5-lemma we see that it is enough to consider one component of $N$. Let $N_{0}$ be a component of $N$ and denote $W \cap N_{0}=W_{0}, C=M-$ $W$ and $C_{0}=C \cap N_{0}=N_{0}-W_{0}$. Using exact homology sequences and the 5-lemma it follows that it is enough to prove that $j_{*}: H_{q}\left(W, W-W_{0}\right) \rightarrow H_{q}\left(M, M-N_{0}\right)$ are isomorphisms for all $q$. We have the commutative diagram

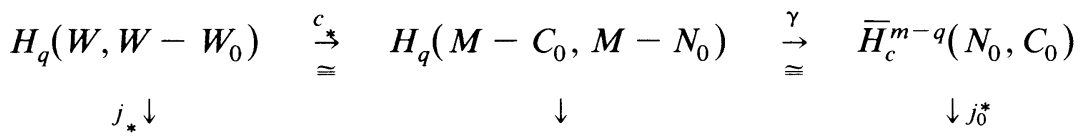

$$
\begin{aligned}
& H_{q}\left(M, M-N_{0}\right) \stackrel{\text { id }}{\stackrel{\longrightarrow}{\cong}} H_{q}\left(M, M-N_{0}\right) \quad \stackrel{\gamma}{\cong} \quad \bar{H}_{c}^{m-q}\left(N_{0}\right)
\end{aligned}
$$

where $m=\operatorname{dim} M$. Here $\bar{H}_{c}^{*}(\mathrm{)})$ denotes Alexander-Spanier cohomology with compact supports and integer coefficients. The maps $\gamma$ are duality isomorphisms, see e.g. Spanier [15, Theorem 6.9.10], and $c_{*}$ is an isomorphism by excision. Since we also have the commutative diagram

$$
\begin{array}{ccc}
H_{p}\left(W_{0}\right)=H_{p}\left(N_{0}-C_{0}\right) & \stackrel{\gamma^{\prime}}{\rightrightarrows} & \bar{H}_{c}^{n-p}\left(N_{0}, C_{0}\right) \\
\left(i_{0}\right)_{*} \downarrow \cong & & \downarrow j_{0}^{*} \\
H_{p}\left(N_{0}\right) & \stackrel{\gamma^{\prime}}{\rightrightarrows} & \bar{H}_{c}^{n-p}\left(N_{0}\right)
\end{array}
$$

where $n=\operatorname{dim} N_{0}$, the proof is complete. 
LEMMA 4.2. Let $M$ denote euclidean space $\mathbf{R}^{m}$ with a smooth action of $G$ such that each fixed-point set $M^{H}$ is homeomorphic to euclidean space $\mathbf{R}^{n(H)}$ and $M^{G} \neq \varnothing$, and $\operatorname{dim} M^{H} \geqslant 4$ for each subgroup $H \neq G$ occurring in $M$. Let $H, H_{1}, \ldots, H_{p}$ be subgroups of $G$ such that $H \subset H_{i}$ and $\operatorname{dim} M^{H_{i}}=\operatorname{dim} M^{H}-1$, for $1 \leqslant i \leqslant p$. Then every component of $M^{H}-\cup_{i=1}^{p^{+}} M^{H_{i}}$ is homeomorphic to euclidean space $\mathbf{R}^{n(H)}$. In particular it follows that if $U$ is a smooth slice at a $\in M^{G}$ the map $i_{\sharp}: \pi_{q}\left(U^{H}-\cup_{i=1}^{p} U^{H_{i}}\right) \rightarrow$ $\pi_{q}\left(M^{H}-\cup_{i=1}^{p} M^{H_{i}}\right)$, induced by the inclusion, is an isomorphism for every $q \geqslant 0$.

Proof. We may assume that the subgroups $H, H_{1}, \ldots, H_{p}$ are such that they occur in their respective fixed-point sets and that $M^{H_{i}} \neq M^{H_{j}}$ for $i \neq j$. Since $M^{H_{i}} \cong$ $\mathbf{R}^{n(H)-1}$ is closed in $M^{H} \cong \mathbf{R}^{n(H)}$ it follows by the Jordan-Brouwer theorem that $M^{H}-M^{H_{i}}$ consists of two components $M_{+}^{i}$ and $M_{-}^{i}, 1 \leqslant i \leqslant p$. Let $U_{+}^{i}$ and $U_{-}^{i}$ be the two components of $U^{H}-U^{H_{i}}$, where $U_{+}^{i} \subset M_{+}^{i}$ and $U_{-}^{i} \subset M_{-}^{i}, 1 \leqslant i \leqslant p$. We now claim the following. Each component of $M^{H}-\cup_{i=1}^{p} M^{H_{i}}$ is of the form $M_{\sigma(1)}^{1} \cap \cdots \cap M_{\sigma(p)}^{p}$, where $\sigma:\{1, \ldots, p\} \rightarrow\{+,-\}$, and each of these components is homeomorphic to $\mathbf{R}^{n(H)}$, and moreover the inclusion $i: U^{H}-\cup_{i=1}^{p} U^{H_{i}} \rightarrow M^{H}-$ $\cup_{i=1}^{p} M^{H_{i}}$ induces a one-to-one correspondence between components. We shall prove this claim by induction in $p$. Since $M^{H_{1}} \cong \mathbf{R}^{n(H)-1}$ and $M^{H_{1}}$ is locally flat and closed in $M^{H} \cong \mathbf{R}^{n(H)}$, where $n(H) \geqslant 4$, it follows by Cantrell [4] (the corollary on p. 718 in [4], see also $\$ 3.3$ in Rushing [13]) that the two components $M_{+}^{1}$ and $M_{-}^{1}$ of $M^{H}-M^{H_{1}}$ are both homeomorphic to $\mathbf{R}^{n(H)}$. Moreover $i: U^{H}-U^{H_{1}} \rightarrow M^{H}-M^{H_{1}}$ induces a one-to-one correspondence of the components.

Now let $p \geqslant 2$ and assume that our claim holds for the values 1 to $p-1$. Observe that in this case $\operatorname{dim} M^{H_{1}} \geqslant 4$, for $\operatorname{dim} M^{H_{1}} \leqslant 3$ would imply $M^{H_{1}}=M^{G}$ and similarly $M^{H_{2}}=M^{G}$, which contradicts the fact that $M^{H_{1}} \neq M^{H_{2}}$. Clearly every component of $M^{H}-\bigcup_{i=1}^{p} M^{H_{i}}$ is contained in some $M_{\sigma(1)}^{1} \cap \cdots \cap M_{\sigma(p)}^{p}$, so we must prove that every nonempty $M_{\sigma(1)}^{1} \cap \cdots \cap M_{\sigma(p)}^{p}$ is connected and homeomorphic to $\mathbf{R}^{n(H)}$. Let $N=M_{\sigma(1)}^{1} \cap \cdots \cap M_{\sigma(p)}^{p} \neq \varnothing$. Then $N^{\prime}=M_{\sigma(1)}^{1} \cap \cdots \cap M_{\sigma(p-1)}^{p-1}$ $\neq \varnothing$ and hence by our inductive assumption $N^{\prime}$ is a component of $M^{H}-\cup_{j=1}^{p-1} M^{H_{j}}$ and $N^{\prime} \cong \mathbf{R}^{n(H)}$. Let us denote $L=M^{H_{p}}$ and let the two components of $M^{H_{p}}-$ $\left(M^{H_{p}} \cap M^{H_{j}}\right)=M^{H_{p}}-M^{\left\langle H_{p}, H_{j}\right\rangle}$ be denoted by $L_{+}^{j}$ and $L_{-}^{j}$, where $L_{+}^{j} \subset M_{+}^{j}$ and $L_{-}^{j} \subset M_{-}^{j}, 1 \leqslant j \leqslant p-1$. Then, by our inductive assumption, each component of $M^{H_{p}}-\bigcup_{j=1}^{p-1} M^{\left\langle H_{p}, H_{j}\right\rangle}$ is of the form $L_{\tau(1)}^{1} \cap \cdots \cap L_{\tau(p-1)}^{p-1}$, where $\tau:\{1, \ldots, p-1\}$ $\rightarrow\{+,-\}$, and each nonempty $L_{\tau(1)}^{1} \cap \cdots \cap L_{\tau(p-1)}^{p-1}$ is homeomorphic to $\mathbf{R}^{n\left(H_{p}\right)}$. We have

$$
\text { (*) } \begin{aligned}
\left(N^{\prime} \cap M_{+}^{p}\right) \dot{U}\left(N^{\prime} \cap M_{-}^{p}\right) & =N^{\prime}-M^{H_{p}}=N^{\prime}-\left(M^{H_{p}}-\bigcup_{j=1}^{p-1} M^{\left\langle H_{p}, H_{j}\right\rangle}\right) \\
& =N^{\prime}-\bigcup_{\tau}\left(L_{\tau(1)}^{1} \cap \cdots \cap L_{\tau(p-1)}^{p-1}\right) \\
& =N^{\prime}-\left(L_{\sigma(1)}^{1} \cap \cdots \cap L_{\sigma(p-1)}^{p-1}\right) .
\end{aligned}
$$

If $L^{\prime}=L_{\sigma(1)}^{1} \cap \cdots \cap L_{\sigma(p-1)}^{p-1}=\varnothing$ we have $N^{\prime} \cap M^{H_{p}}=\varnothing$ and hence, since $N^{\prime}$ is connected, either $N^{\prime} \subset M_{+}^{p}$ or $N^{\prime} \subset M_{-}^{p}$. Since $N=N^{\prime} \cap M_{\sigma(p)}^{p} \neq \varnothing$ we have 
$N^{\prime} \subset M_{\sigma(p)}^{p}$, and hence in this case $N=N^{\prime} \cap M_{\sigma(p)}^{p}=N^{\prime}$, which is connected and homeomorphic to $\mathbf{R}^{n(H)}$. If $L^{\prime} \neq \varnothing$ we have $N^{\prime} \cap M^{H_{p}} \neq \varnothing$, and hence $N^{\prime} \cap M_{+}^{p}$ $\neq \varnothing$ and $N^{\prime} \cap M_{-}^{p} \neq \varnothing$. Since $L^{\prime} \subset N^{\prime}$ and $L^{\prime} \cong \mathbf{R}^{n(H)-1}$ is locally flat and closed in $N^{\prime} \cong \mathbf{R}^{n(H)}$ it again follows by Cantrell [4] that the two components of $N^{\prime}-L^{\prime}$ are both homeomorphic to $\mathbf{R}^{n(H)}$. Since (*) implies that the two components of $N^{\prime}-L^{\prime}$ are $N^{\prime} \cap M_{+}^{p}$ and $N^{\prime} \cap M_{-}^{p}$, it now follows that $N=N^{\prime} \cap M_{\sigma(p)}^{p}$ is homeomorphic to $\mathbf{R}^{n(H)}$.

The map between the sets of components of $U^{H}-\cup_{i=1}^{p} U^{H_{i}}$ and $M^{H}-$ $\cup_{i=1}^{p} M^{H_{i}}$, induced by the inclusion, assigns to a (nonempty) component $U_{\sigma(1)}^{1}$ $\cap \cdots \cap U_{\sigma(p)}^{p}$ the component $M_{\sigma(1)}^{1} \cap \cdots \cap M_{\sigma(p)}^{p}$ and hence this map is clearly injective. It remains to show that it is surjective, namely that $N=M_{\sigma(1)}^{1}$ $\cap \cdots \cap M_{\sigma(p)}^{p} \neq \varnothing$ implies $V=U_{\sigma(1)}^{1} \cap \cdots \cap U_{\sigma(p)}^{p} \neq \varnothing$. Since $N^{\prime}=M_{\sigma(1)}^{1}$ $\cap \cdots \cap M_{\sigma(p-1)}^{p-1} \neq \varnothing$ we have by the inductive assumption that $V^{\prime}=U_{\sigma(1)}^{1}$ $\cap \cdots \cap U_{\sigma(p-1)}^{p(p-1)} \neq \varnothing$. Let $W_{+}^{j}$ and $W_{-}^{j}$ be the two components of $U^{H_{p}}-U^{\left\langle H_{p}, H_{j}\right\rangle}$, where $W_{+}^{j} \subset L_{+}^{j}$ and $W_{-}^{j} \subset L_{-}^{j}, 1 \leqslant j \leqslant p-1$. Then we get, in analogy with (*),

$(* *) \quad\left(V^{\prime} \cap U_{+}^{p}\right) \dot{U}\left(V^{\prime} \cap U_{-}^{p}\right)=V^{\prime}-U^{H_{p}}=V^{\prime}-\left(W_{\sigma(1)}^{1} \cap \cdots \cap W_{\sigma(p-1)}^{p-1}\right)$.

If $L^{\prime}=\varnothing$ we have, as we saw above, that $N^{\prime} \subset M_{\sigma(p)}^{p}$. Therefore $V^{\prime} \subset N^{\prime} \subset M_{\sigma(p)}^{p}$ and $V^{\prime} \subset M_{\sigma(p)}^{p} \cap U=U_{\sigma(p)}^{p}$, and hence $V=V^{\prime} \cap U_{\sigma(p)}^{p}=V^{\prime} \neq \varnothing$. If $L^{\prime} \neq \varnothing$ it follows by the inductive assumption that $W^{\prime}=W_{\sigma(1)}^{1} \cap \cdots \cap W_{\sigma(p-1)}^{p-1} \neq \varnothing$, and hence by (**) we have that $V^{\prime} \cap U^{H_{p}} \neq \varnothing$, which in turn implies that both $V^{\prime} \cap U_{+}^{p} \neq \varnothing$ and $V^{\prime} \cap U_{-}^{p} \neq \varnothing$. Thus $V=V^{\prime} \cap U_{\sigma(p)}^{p} \neq \varnothing$. This completes the induction and the proof of Lemma 4.2.

LEMMA 4.3. Let $M$ be as in 4.2, and let $H, H_{1}$ and $H_{2}$ be subgroups of $G$, where $H \subsetneq H_{1}$ and $H \subsetneq H_{2}$, such that $\operatorname{dim} M^{H_{1}}=\operatorname{dim} M^{H}-1$ and $\operatorname{dim} M^{H_{2}}=\operatorname{dim} M^{H}$ 2. Let $U$ be a smooth slice at a point $a \in M^{G}$ and assume that, for any subgroups $K \subsetneq K_{2}$ with $\operatorname{dim} M^{K_{2}}=\operatorname{dim} M^{K}-2$, the map

$$
i_{\sharp}^{\prime}: \pi_{q}\left(U^{K}-U^{K_{2}}\right) \rightarrow \pi_{q}\left(M^{K}-M^{K_{2}}\right),
$$

induced by the inclusion $i^{\prime}$, is an isomorphism for every $q \geqslant 1$. Then the map $j_{\sharp}: \pi_{q}\left(U^{H}-\left(U^{H_{1}} \cup U^{H_{2}}\right)\right) \rightarrow \pi_{q}\left(M^{H}-\left(M^{H_{1}} \cup M^{H_{2}}\right)\right)$, induced by the inclusion $j$, is an isomorphism for every $q \geqslant 0$.

Proof. Since $M^{H_{1}}$ is closed in $M^{H}$ it follows by the Jordan-Brouwer separation theorem that $M^{H}-M^{H_{1}}$ consists of two components $M_{+}$and $M_{-}$such that $\bar{M}_{+} \cup \bar{M}_{-}=M^{H}$ and $\bar{M}_{+} \cap \bar{M}_{-}=M^{H_{1}}$. Likewise $U^{H}-U^{H_{1}}$ consists of two components $U_{+}$and $U_{-}$, where $U_{+} \subset M_{+}$, such that $\bar{U}_{+} \cup \bar{U}_{-}=U^{H}$ and $\bar{U}_{+} \cap \bar{U}_{-}=U^{H_{1}}$. The spaces $U_{+}-U^{H_{2}}$ and $M_{+}-M^{H_{2}}$ are connected, and we shall prove that

$$
j_{\sharp}: \pi_{q}\left(U_{+}-U^{H_{2}}\right) \rightarrow \pi_{q}\left(M_{+}-M^{H_{2}}\right)
$$

is an isomorphism for every $q \geqslant 1$. The proof in the case of the minus-components is of course completely analogous.

If $U^{H_{2}} \subset U^{H_{1}}$ we also have $M^{H_{2}} \subset M^{H_{1}}$ and hence in this case $U_{+}-U^{H_{2}}=U_{+}$ and $M_{+}-M^{H_{2}}=M_{+}$. Since $M^{H_{1}} \cong \mathbf{R}^{n(H)-1}$ is locally flat and closed in $M^{H} \cong \mathbf{R}^{n(H)}$ it follows by Cantrell [4] that $M_{+}$is homeomorphic to $\mathbf{R}^{n(H)}$. Since also $U_{+} \cong \mathbf{R}^{n(H)}$ our claim is clear in this case. 
Let us now assume that $U^{H_{2}} \not \subset U^{H_{1}}$. Let $\alpha^{\prime}: \mathbf{R}^{m}(\rho) \rightarrow U$ be a $G$-diffeomorphism, and denote $n=\operatorname{dim}\left(\mathbf{R}^{m}(\rho)\right)^{H}=\operatorname{dim} U^{H}$. Then $\operatorname{dim}\left(\mathbf{R}^{m}(\rho)\right)^{H_{1}}=n-1$, $\operatorname{dim}\left(\mathbf{R}^{m}(\rho)\right)^{H_{2}}=n-2$ and $\left(\mathbf{R}^{m}(\rho)\right)^{H_{2}} \not \subset\left(\mathbf{R}^{m}(\rho)\right)^{H_{1}}$. By a linear change of coordinates in $\mathbf{R}^{n}=\left(\mathbf{R}^{m}(\rho)\right)^{H}$ we get the situation that

$$
\alpha=\alpha^{\prime} \mid: \mathbf{R}^{n} \stackrel{\cong}{\rightarrow} U^{H}
$$

is a homeomorphism such that

$$
\begin{aligned}
& \alpha\left(\mathbf{R}^{n-1}\right)=U^{H_{1}}, \\
& \alpha\left(\hat{\mathbf{R}}^{n-2}\right)=U^{H_{2}}, \\
& \alpha\left(\mathbf{R}_{+}^{n}\right)=\bar{U}_{+} \quad(\text { here the closure is with respect to } U),
\end{aligned}
$$

where $\mathbf{R}^{n-1}=\mathbf{R}^{n-1} \times\{0\} \subset \mathbf{R}^{n}, \hat{\mathbf{R}}^{n-2}=\mathbf{R}^{n-3} \times\{0\} \times\{0\} \times \mathbf{R} \subset \mathbf{R}^{n}$ and $\mathbf{R}_{+}^{n}=$ $\left\{\left(x_{1}, \ldots, x_{n}\right) \in \mathbf{R}^{n} \mid x_{n} \geqslant 0\right\}$. For $X \subset \mathbf{R}^{n}$ we set $B(X)=\{x \in X \mid\|x\| \leqslant 1\}$, and denote $B\left(\mathbf{R}^{n}\right)=B^{n}, B\left(\mathbf{R}^{n-1}\right)=B^{n-1}, B\left(\hat{\mathbf{R}}^{n-2}\right)=\hat{B}^{n-2}$ and $B\left(\mathbf{R}_{+}^{n}\right)=B_{+}^{n}$. Now define

$$
\begin{aligned}
& \alpha\left(B^{n}\right)=A^{n} \subset U^{H}, \\
& \alpha\left(B^{n-1}\right)=A^{n-1} \subset U^{H_{1}}, \\
& \alpha\left(\hat{B}^{n-2}\right)=\hat{A}^{n-2} \subset U^{H_{2}}, \\
& \alpha\left(B_{+}^{n}\right)=A_{+}^{n} \subset \bar{U}_{+} .
\end{aligned}
$$

Since the inclusions $B^{n}-\hat{B}^{n-2} \hookrightarrow \mathbf{R}^{n}-\hat{\mathbf{R}}^{n-2}$ and $B_{+}^{n}-\hat{B}^{n-2} \hookrightarrow \mathbf{R}_{+}^{n}-\hat{\mathbf{R}}^{n-2}$ are homotopy equivalences it follows that the inclusions

$$
\begin{gathered}
A^{n}-\hat{A}^{n-2} \hookrightarrow U^{H}-U^{H_{2}}, \\
A_{+}^{n}-\hat{A}^{n-2} \hookrightarrow \bar{U}_{+}-U^{H_{2}}
\end{gathered}
$$

are homotopy equivalences. By (1) and our assumption, it follows that

$$
i_{\sharp}: \pi_{q}\left(A^{n}-\hat{A}^{n-2}\right) \stackrel{\cong}{\rightarrow} \pi_{q}\left(M^{H}-M^{H_{2}}\right)
$$

is an isomorphism for every $q \geqslant 1$. Now $A^{n}-\hat{A}^{n-2}$ is a smooth submanifold, with boundary, of $M^{H}-M^{H_{2}}$. The boundary $\partial\left(A^{n}-\hat{A}^{n-2}\right)$ is closed in $M^{H}-M^{H_{2}}$ and $\left(M^{H}-M^{H_{2}}\right)-\partial\left(A^{n}-\hat{A}^{n-2}\right)$ consists of two components. Hence it follows that $M^{H}-M^{H_{2}}$ can be triangulated in such a way that $A^{n}-\hat{A}^{n-2}$ is a subcomplex of $M^{H}-M^{H_{2}}$. Since (3) holds it now follows that $A^{n}-\hat{A}^{n-2}$ is a strong deformation retract of $M^{H}-M^{H_{2}}$. Let

$$
F:\left(M^{H}-M^{H_{2}}\right) \times I \rightarrow M^{H}-M^{H_{2}}
$$

be a homotopy $\operatorname{rel}\left(A^{n}-\hat{A}^{n-2}\right)$ from the identity to the map $i \circ r$, where

$$
r: M^{H}-M^{H_{2}} \rightarrow A^{n}-\hat{A}^{n-2}
$$

is a retraction and $i: A^{n}-\hat{A}^{n-2} \hookrightarrow M^{H}-M^{H_{2}}$ is the inclusion.

We have $M^{H_{1}} \cap M^{H_{2}}=M^{H_{3}}$, where $H_{3}=\left[H_{1}, H_{2}\right]$ denotes the subgroup generated by $H_{1}$ and $H_{2}$, and since $\operatorname{dim} M^{H_{3}}=n-3$ we have by assumption that $i_{\sharp}^{\prime}$ : $\pi_{q}\left(U^{H_{1}}-U^{H_{3}}\right) \rightarrow \pi_{q}\left(M^{H_{1}}-M^{H_{3}}\right)$ is an isomorphism for all $q \geqslant 1$. Therefore it follows, in the same way as above, that $A^{n-1}-A^{n-3}$ is a strong deformation retract of $M^{H_{1}}-M^{H_{3}}$, where $A^{n-3}=A^{n-1} \cap \hat{A}^{n-2}$. Hence $A^{n}-\hat{A}^{n-2}$ is a strong deformation retract of $\left(A^{n}-\hat{A}^{n-2}\right) \cup\left(M^{H_{1}}-M^{H_{3}}\right)$, and since the corresponding inclusion as well as the inclusion $i$ are homotopy equivalences it follows that the inclusion 
$\left(A^{n}-\hat{A}^{n-2}\right) \cup\left(M^{H_{1}}-M^{H_{3}}\right) \hookrightarrow M^{H}-M^{H_{2}}$ is a homotopy equivalence. Thus $\left(\hat{A}-\hat{A}^{n-2}\right) \cup\left(M^{H_{1}}-M^{H_{3}}\right)$ is a strong deformation retract of $M^{H}-M^{H_{2}}$, and in particular there exists a retraction

$$
\bar{r}: M^{H}-M^{H_{2}} \rightarrow\left(A^{n}-\hat{A}^{n-2}\right) \cup\left(M^{H_{1}}-M^{H_{3}}\right) .
$$

Let

$$
r_{0}^{\prime}: B^{n}-\hat{B}^{n-2} \rightarrow B_{+}^{n}-\hat{B}^{n-2}
$$

be the retraction defined by

$$
\left\{\begin{array}{l}
r_{0}^{\prime} \mid B_{+}^{n}-B^{n-2}=\mathrm{id}, \\
r_{0}^{\prime}\left(x_{1}, \ldots, x_{n}\right)=\left(x_{1}, \ldots,-x_{n}\right) \text { for }\left(x_{1}, \ldots, x_{n}\right) \in B_{-}^{n}-\hat{B}^{n-2} .
\end{array}\right.
$$

Then

$$
r_{0}=\alpha \circ r_{0}^{\prime} \circ \alpha^{-1}: A^{n}-\hat{A}^{n-2} \rightarrow A_{+}^{n}-\hat{A}^{n-2}
$$

is a retraction, and extending by the identity map we get a retraction

$$
\bar{r}_{0}:\left(A^{n}-\hat{A}^{n-2}\right) \cup\left(M^{H_{1}}-M^{H_{3}}\right) \rightarrow\left(A_{+}^{n}-\hat{A}^{n-2}\right) \cup\left(M^{H_{1}}-M^{H_{3}}\right) .
$$

We now define a retraction

$$
\tilde{r}: M^{H}-M^{H_{2}} \rightarrow \bar{M}_{+}-M^{H_{2}}
$$

by

$$
\left\{\begin{array}{l}
\tilde{r} \mid \bar{M}_{+}-M^{H_{2}}=\mathrm{id} \\
\tilde{r}\left|\bar{M}_{-}-M^{H_{2}}=\left(\bar{r}_{0} \circ \bar{r}\right)\right|\left(\bar{M}_{-}-M^{H_{2}}\right) .
\end{array}\right.
$$

Then the composite

$$
\left(\bar{M}_{+}-M^{H_{2}}\right) \times I \stackrel{i \times \text { id }}{\hookrightarrow}\left(M^{H}-M^{H_{2}}\right) \times I \stackrel{F}{\rightarrow} M^{H}-M^{H_{2}} \stackrel{\tilde{r}}{\rightarrow} \bar{M}_{+}-M^{H_{2}}
$$

is a homotopy $\operatorname{rel}\left(A_{+}^{n}-\hat{A}^{n-2}\right)$ from the identity to $i_{+} \circ r_{0} \circ r \circ \tilde{i}$, where $i_{+}: A_{+}^{n}-$ $\hat{A}^{n-2} \hookrightarrow \bar{M}_{+}-M^{H_{2}}$ and $\tilde{i}: \bar{M}_{+}-M^{H_{2}} \hookrightarrow M^{H}-M^{H_{2}}$ denote inclusions, and $r_{0} \circ r \circ \tilde{i}: \bar{M}_{+}-M^{H_{2}} \rightarrow A_{+}^{n}-\hat{A}^{n-2}$ is a retraction. Thus

$$
\left(i_{+}\right)_{\sharp}: \pi_{q}\left(A_{+}^{n}-\hat{A}^{n-2}\right) \stackrel{\cong}{\rightarrow} \pi_{q}\left(\bar{M}_{+}-M^{H_{2}}\right)
$$

is an isomorphism for every $q$. Together with (2) this now implies that

$$
\overline{j_{\#}}: \pi_{q}\left(\bar{U}_{+}-U^{H_{2}}\right) \stackrel{\cong}{\rightarrow} \pi_{q}\left(\bar{M}_{+}-M^{H_{2}}\right)
$$

is an isomorphism for all $q$. Since $\bar{M}_{+}-M^{H_{2}}$ is a manifold with boundary whose interior is $M_{+}-M^{H_{2}}$ it follows, using a collar of the boundary, that the inclusion $M_{+}-M^{H_{2}} \hookrightarrow \bar{M}_{+}-M^{H_{2}}$ is a homotopy equivalence. Also $U_{+}-U^{H_{2}} \hookrightarrow \bar{U}_{+}-U^{H_{2}}$ is a homotopy equivalence and hence

$$
j_{\sharp}: \pi_{q}\left(U_{+}-U^{H_{2}}\right) \stackrel{\cong}{\rightarrow} \pi_{q}\left(M_{+}-M^{H_{2}}\right)
$$

is an isomorphism for all $q$. 
Lemma 4.4. Assume that $G$ is a finite abelian group, and let $M$ be as in 4.2. Let $H$ be a subgroup of $G$, and let $H_{1}, \ldots, H_{p}$ and $K_{1}, \ldots, K_{r}$ be subgroups of $G$ containing $H$ such that $\operatorname{dim} M^{H_{i}}=\operatorname{dim} M^{H}-1,1 \leqslant i \leqslant p$, and $\operatorname{dim} M^{K_{i}}=\operatorname{dim} M^{H}-2$, $1 \leqslant i \leqslant r$. Let $U$ be a smooth slice at a point $a \in M^{G}$ and assume that the map $i_{\sharp}: \pi_{q}\left(U^{H}-\cup_{i=1}^{r} U^{K_{i}}\right) \rightarrow \pi_{q}\left(M^{H}-\cup_{i=1}^{r} M^{K_{i}}\right)$, induced by the inclusion $i$, is an isomorphism for every $q \geqslant 1$. Then the map

$$
j_{\sharp}: \pi_{q}\left(U^{H}-\left(\bigcup_{i=1}^{p} U^{H_{i}} \cup \bigcup_{i=1}^{r} U^{K_{i}}\right)\right) \rightarrow \pi_{q}\left(M^{H}-\left(\bigcup_{i=1}^{p} M^{H_{i}} \cup \bigcup_{i=1}^{r} M^{K_{i}}\right)\right) \text {, }
$$

induced by the inclusion $j$, is an isomorphism for every $q \geqslant 0$.

Proof. First of all we may assume that $M^{H_{i}} \neq M^{H_{j}}$, if $i \neq j$. Furthermore we may assume that all the groups $H, H_{1}, \ldots, H_{p}$ and $K_{1}, \ldots, K_{r}$ are chosen such that they occur in their respective fixed-point sets. Since $M^{H_{i}}$ is closed in $M^{H}$ it follows by the Jordan-Brouwer separation theorem that $M^{H}-M^{H_{i}}$ consists of two components $M_{+}^{i}$ and $M_{-}^{i}$ such that $\bar{M}_{+}^{i} \cup \bar{M}_{-}^{i}=M^{H}$ and $\bar{M}_{+}^{i} \cap \bar{M}_{-}^{i}=M^{H_{i}}, 1 \leqslant i \leqslant p$. Likewise $U^{H}-U^{H_{i}}$ consists of two components $U_{+}^{i}$ and $U_{-}^{i}$, where $U_{+}^{i} \subset M_{+}^{i}$, such that $\bar{U}_{+}^{i} \cup \bar{U}_{-}^{i}=U^{H}$ and $\bar{U}_{+}^{i} \cap \bar{U}_{-}^{i}=U^{H_{i}}, 1 \leqslant i \leqslant p$.

Since $G$ is abelian any $g \in G$ induces a homeomorphism $g: M^{H} \rightarrow M^{H}$, and if $g \in H_{i}$ we have $g \mid M^{H_{i}}=$ id. We now claim that for any $g_{i} \in H_{i} \backslash H$ we have

$$
\left\{\begin{array}{l}
g_{i}\left(U_{+}^{i}\right)=U_{-}^{i}, \\
g_{i}\left(U_{-}^{i}\right)=U_{+}^{i},
\end{array} \quad 1 \leqslant i \leqslant p\right.
$$

and

$$
\left\{\begin{array}{l}
g_{i}\left(M_{+}^{i}\right)=M_{-}^{i}, \\
g_{i}\left(M_{-}^{i}\right)=M_{+}^{i},
\end{array} \quad 1 \leqslant i \leqslant p\right.
$$

To see this let $\alpha: \mathbf{R}^{m}(\rho) \rightarrow U$ be a $G$-homeomorphism. Then $\left(\mathbf{R}^{m}(\rho)\right)^{H}=\mathbf{R}^{n}(\tau)$ is a representation space for $H_{i}$ and $\alpha\left(\mathbf{R}^{n}(\tau)\right)=U^{H}$. Furthermore $\mathbf{R}^{n}(\tau)=\mathbf{R}^{n-1} \oplus \mathbf{R}\left(\tau^{\prime}\right)$, where $H_{i}$ acts trivially on $\mathbf{R}^{n-1}$. If the action of $g_{i}$ on $\mathbf{R}\left(\tau^{\prime}\right)$ is trivial we would have $\left(\mathbf{R}^{n}(\tau)\right)^{\left\langle H,\left\langle g_{i}\right\rangle\right\rangle}=\mathbf{R}^{n}(\tau)$ and hence $U^{\left\langle H,\left\langle g_{i}\right\rangle\right\rangle}=U^{H}$. But since $H$ occurs in $M$ and hence also in $U$ and $H \subsetneq\left\langle H,\left\langle g_{i}\right\rangle\right\rangle$, this is a contradiction. Hence $g_{i}$ acts on $\mathbf{R}\left(\tau^{\prime}\right)$ by $x \mapsto-x$ and it follows that (1) and (2) hold.

Next we claim that

$$
\left\{\begin{array}{l}
g_{i}\left(M_{+}^{j}\right)=M_{+}^{j}, \\
g_{i}\left(M_{-}^{j}\right)=M_{-}^{j},
\end{array} \quad 1 \leqslant j \leqslant p \text { and } j \neq i\right.
$$

and

$$
\left\{\begin{array}{l}
g_{i}\left(U_{+}^{j}\right)=U_{+}^{j}, \\
g_{i}\left(U_{-}^{j}\right)=U_{-}^{j}
\end{array} \quad 1 \leqslant j \leqslant p \text { and } j \neq i\right.
$$


This is seen as follows. Since $M^{H_{i}} \neq M^{H_{j}}$, and $M^{H_{i}}$ and $M^{H_{j}}$ are connected and closed in $M^{H}$, it follows that $M^{H_{i}} \cap M^{H_{j}}=M^{\left\langle H_{i}, H_{j}\right\rangle}$ is $(n-2)$-dimensional. Choose $y \in M^{H_{i}}-M^{\left\langle H_{i}, H_{j}\right\rangle} \neq \varnothing$. Then $y \in M^{H}-M^{H_{j}}=M_{+}^{j} \dot{\cup} M_{-}^{j}$. Because $G$ is abelian we have the homeomorphism $g_{i}: M^{H}-M^{H_{j}} \rightarrow M^{H}-M^{H_{j}}$. Since $g_{i}(y)=y$ it now follows that $g_{i}\left(M_{+}^{j}\right)=M_{+}^{j}$ and $g_{i}\left(M_{-}^{j}\right)=M_{-}^{j}$. This proves (3) and (4).

As we showed in the proof of Lemma 4.2 any component of $M^{H}-\cup_{i=1}^{p} M^{H_{i}}$ is of the form $M_{\sigma(1)}^{1} \cap \cdots \cap M_{\sigma(p)}^{p}$, where $\sigma:\{1, \ldots, p\} \rightarrow\{+,-\}$, and moreover the inclusion $U^{H}-\cup_{i=1}^{p} U^{H_{i}} \hookrightarrow M^{H}-\bigcup_{i=1}^{p} M^{H_{i}}$ induces a one-to-one correspondence between the components. In fact, since at least one of the intersections $M_{\sigma(1)}^{1}$ $\cap \cdots \cap M_{\sigma(p)}^{p}$ must be nonempty, it follows by (2) and (3) that all $2^{p}$ of them are nonempty. Likewise every component of $U^{H}-\bigcup_{i=1}^{p} U^{H_{i}}$ is of the form $U_{\sigma(1)}^{1}$ $\cap \cdots \cap U_{\sigma(p)}^{p}$, and there are $2^{p}$ of them. Since $\operatorname{dim} M^{K_{i}}=\operatorname{dim} M^{H}-2,1 \leqslant i \leqslant r$, it follows that each component of $M^{H}-\left(\cup_{i=1}^{p} M^{H_{i}} \cup \cup_{i=1}^{r} M^{K_{i}}\right)$ is of the form $N-\cup_{i=1}^{r} M^{K_{i}}$, where $N$ is a component of $M^{H}-\cup_{i=1}^{p} M^{H_{i}}$, and analogously for $U$. Hence it follows that the inclusion

$$
j: U^{H}-\left(\bigcup_{i=1}^{p} U^{H_{i}} \cup \bigcup_{i=1}^{r} U^{K_{i}}\right) \rightarrow M^{H}-\left(\bigcup_{i=1}^{p} M^{H_{i}} \cup \bigcup_{i=1}^{r} M^{K_{i}}\right)
$$

induces a one-to-one correspondence between the components. Let us now denote $V=U_{+}^{1} \cap \cdots \cap U_{+}^{p}$ and $N=M_{+}^{1} \cap \cdots \cap M_{+}^{p}$. Our claim is that

$$
j_{\#}: \pi_{q}\left(V-\bigcup_{i=1}^{r} U^{K_{i}}\right) \rightarrow \pi_{q}\left(N-\bigcup_{i=1}^{r} M^{K_{i}}\right)
$$

is an isomorphism for every $q \geqslant 1$. The proof of this fact for any other pair of components is of course completely similar, and in fact using (1)-(4) it follows that the validity of the case in (5) implies the validity of all the other cases.

First of all we claim that

$$
\bar{N}=\bar{M}_{+}^{1} \cap \cdots \cap \bar{M}_{+}^{p},
$$

and that $\bar{N}$ is a topological manifold with boundary whose interior equals $N$. This is seen as follows. The inclusion $\bar{N} \subset \cup_{i=1}^{p} \bar{M}_{+}^{i}$ is clear. Now let $y \in \cap_{i=1}^{p} \bar{M}_{+}^{i}$. We may assume that $y \in \bigcap_{i=1}^{t} M^{H_{i}} \cap \bigcap_{i=t+1}^{p} M_{+}^{i}$, where $1 \leqslant t \leqslant p$. Let $W_{y}$ be a smooth slice at $y$ in the smooth $G$-manifold $M^{H}$. Then the pair $\left(W_{y}, \cup_{i=1}^{t} W_{y}^{H_{i}}\right)$ is homeomorphic to a pair $\left(\mathbf{R}^{n}(\tau), \cup_{i=1}^{t} \mathbf{R}^{n}(\tau)^{H_{i}}\right)$, where $\tau: G_{y} \rightarrow O(n)$. Thus we see that $y$ belongs to the closure of each of the $2^{t}$ components of $W_{y}-\cup_{i=1}^{t} W_{y}^{H_{i}}$, and hence it follows that $y \in \bigcap_{i=1}^{t} \bar{M}_{+}^{i}$ and therefore $y \in \cap_{i=1}^{t} \vec{M}_{+}^{i} \cap \bigcap_{i=t+1}^{p} M_{+}^{i} \subset$ $\cap_{i=1}^{p} \bar{M}_{+}^{i}$. Thus (6) is valid. Moreover the neighbourhood $W_{y} \cap \bar{N}$ of $y$ in $\bar{N}$ is homeomorphic to a set of the form $\left\{\left(x_{1}, \ldots, x_{n}\right) \in \mathbf{R}^{n} \mid x_{n-t+1} \geqslant 0, \ldots, x_{n} \geqslant 0\right\}$. Hence $\bar{N}$ is a topological manifold with boundary and $\partial \bar{N}=\bar{N}-N$.

Similarly we have $\bar{V}=\bar{U}_{+}^{1} \cap \cdots \cap \bar{U}_{+}^{p}$ and $\bar{V}$ is a topological manifold with boundary whose interior equals $V$. Since $\bar{N}-\cup_{i=1}^{r} M^{K_{i}}$ is open in $\bar{N}$ it follows that $\bar{N}-\cup_{i=1}^{r} M^{K_{i}}$ is a topological manifold with boundary whose interior equals $N-\cup_{i=1}^{r} M^{K_{i}}$. Similarly $\bar{V}-\cup_{i=1}^{r} U^{K_{i}}$ is a topological manifold with boundary 
whose interior equals $V-\cup_{i=1}^{r} U^{K_{i}}$. Hence it follows (by using a collar of the boundary) that the inclusions

$$
\left\{\begin{array}{l}
V-\bigcup_{i=1}^{r} U^{K_{i}} \hookrightarrow \bar{V}-\bigcup_{i=1}^{r} U^{K_{i}} \\
N-\bigcup_{i=1}^{r} M^{K_{i}} \hookrightarrow \bar{N}-\bigcup_{i=1}^{r} M^{K_{i}}
\end{array}\right.
$$

are homotopy equivalences. Therefore in order to prove that (5) is an isomorphism for every $q \geqslant 1$ it is enough to prove that

$$
\overline{j_{\sharp}}: \pi_{q}\left(\bar{V}-\bigcup_{i=1}^{r} U^{K_{i}}\right) \rightarrow \pi_{q}\left(\bar{N}-\bigcup_{i=1}^{r} M^{K_{i}}\right)
$$

is an isomorphism for every $q \geqslant 1$.

We shall now conclude the proof of the lemma by showing that (8) is an isomorphism for every $q \geqslant 1$. Choose $g_{i} \in H_{i} \backslash H, i=1, \ldots, p$. Define a retraction

$$
r_{1}: M^{H}-\bigcup_{i=1}^{r} M^{K_{i}} \rightarrow \bar{M}_{+}^{1}-\bigcup_{i=1}^{r} M^{K_{i}}
$$

by

$$
\begin{cases}r_{1}(x)=x, & x \in \bar{M}_{+}^{1}-\bigcup_{i=1}^{r} M^{K_{i}}, \\ r_{1}(x)=g_{1}(x), & x \in \bar{M}_{-}^{1}-\bigcup_{i=1}^{r} M^{K_{i}} .\end{cases}
$$

Since $G$ is abelian we have $g_{1}\left(M^{K_{i}}\right)=M^{K_{i}}, 1 \leqslant i \leqslant r$, and it follows that $r_{1}$ is a well-defined retraction. Moreover $r_{1}$ restricts to give a retraction

$$
r_{1} \mid: U^{H}-\bigcup_{i=1}^{r} U^{K_{i}} \rightarrow \bar{U}_{+}^{1}-\bigcup_{i=1}^{r} U^{K_{i}}
$$

Now we define

$$
r_{2}: \bar{M}_{+}^{1}-\bigcup_{i=1}^{r} M^{K_{i}} \rightarrow\left(\bar{M}_{+}^{1} \cap \bar{M}_{+}^{2}\right)-\bigcup_{i=1}^{r} M^{K_{i}}
$$

by

$$
\begin{cases}r_{2}(x)=x, & x \in\left(\bar{M}_{+}^{1} \cap \bar{M}_{+}^{2}\right)-\bigcup_{i=1}^{r} M^{K_{i}}, \\ r_{2}(x)=g_{2}(x), & x \in\left(\bar{M}_{+}^{1} \cap \bar{M}_{-}^{2}\right)-\bigcup_{i=1}^{r} M^{K_{i}} .\end{cases}
$$

Then $r_{2}$ restricts to a retraction $r_{2} \mid: \bar{U}_{+}^{1}-\cup_{i=1}^{r} U^{K_{i}} \rightarrow\left(\bar{U}_{+}^{1} \cap \bar{U}_{+}^{2}\right)-\cup_{i=1}^{r} U^{K_{i}}$. Continuing in this way we get a retraction

$$
R=r_{p} \circ \cdots \circ r_{1}: M^{H}-\bigcup_{i=1}^{r} M^{K_{i}} \rightarrow \bar{N}-\bigcup_{i=1}^{r} M^{K_{i}},
$$


such that also

$$
R \mid: U^{H}-\bigcup_{i=1}^{r} U^{K_{i}} \rightarrow \bar{V}-\bigcup_{i=1}^{r} U^{K_{i}}
$$

is a retraction. Now consider the following diagram:

$$
\begin{array}{ccc}
\pi_{q}\left(U^{H}-\bigcup_{i=1}^{r} U^{K_{i}}\right) & \stackrel{i_{\sharp}}{\rightarrow} \pi_{q}\left(M^{H}-\bigcup_{i=1}^{r} M^{K_{i}}\right) \\
\pi_{q}\left(\bar{V}-\bigcup_{i=1}^{r} U^{K_{i}}\right) & \stackrel{\bar{j}_{\sharp}}{\rightarrow} & \pi_{q}\left(\bar{N}-\bigcup_{i=1}^{r} M^{i_{i}}\right) \\
& &
\end{array}
$$

Here $i_{\sharp}$ is an isomorphism by assumption. The maps $i_{\sharp}^{\prime}$ and $i_{\#}^{\prime \prime}$ are injective and $(R \mid)_{\sharp}$ and $R_{\sharp}$ are surjective. Since $i_{\#}^{\prime \prime} \circ \overline{j_{\sharp}}=i_{\sharp} \circ i_{\sharp}^{\prime}$ is injective it follows that $\overline{j_{\sharp}}$ is injective. Since $\bar{j}_{\sharp} \circ(R \mid)_{\sharp}=R_{\sharp} \circ i_{\sharp}$ is surjective it follows that $\overline{j_{\sharp}}$ is surjective. Hence $\overline{j_{\sharp}}$ is an isomorphism for all $q \geqslant 1$.

LEMMA 4.5. Let $M$ be as in 4.2, and assume that either:

(A) Any simultaneous codimension 1 and 2 fixed-point situation is simple, or

(B) $G$ is abelian.

Let $U$ be a smooth slice at a point $a \in M^{G}$ and assume that for any subgroup $H$ of $G$ and any subgroups $K_{i} \supsetneq H$ such that $\operatorname{dim} M^{K_{i}}=M^{H}-2,1 \leqslant i \leqslant r$, the map

$$
i_{\sharp}: \pi_{q}\left(U^{H}-\bigcup_{i=1}^{r} U^{K_{i}}\right) \rightarrow \pi_{q}\left(M^{H}-\bigcup_{i=1}^{r} M^{K_{i}}\right) \text {, }
$$

induced by the inclusion $i$, is an isomorphism for every $q \geqslant 1$. Then the map $j_{\sharp}: \pi_{q}\left(U_{H}\right)$ $\rightarrow \pi_{q}\left(M_{H}\right)$, induced by the inclusion $j$, is an isomorphism for every subgroup $H$ and every $q \geqslant 0$.

Proof. For any subgroup $H$ of $G$ we define "Assertion $H$ " as follows.

Assertion $H$ : For any subgroups $H_{1}, \ldots, H_{s}$ of $G$ such that $H_{i} \supsetneq H, 1 \leqslant i \leqslant s$, the map

$$
j_{\sharp}: \pi_{q}\left(U^{H}-\bigcup_{i=1}^{s} U^{H_{i}}\right) \rightarrow \pi_{q}\left(M^{H}-\bigcup_{i=1}^{s} M^{H_{i}}\right),
$$

induced by the inclusion $j$, is an isomorphism for every $q \geqslant 0$.

Now let $H$ be an arbitrary subgroup of $G$ and assume inductively that "Assertion $K$ " is valid for all subgroups $K$ of $G$ that are strictly greater than $H$. Observe that "Assertion $G$ " holds. We shall prove that "Assertion $H$ " is valid. Let $H_{1}, \ldots, H_{s}$ be any subgroups of $G$ such that $H_{i} \supsetneq H$ and $\operatorname{dim} M^{H_{i}} \leqslant \operatorname{dim} M^{H}-1,1 \leqslant i \leqslant s$. (If $\operatorname{dim} M^{H_{i}}=\operatorname{dim} M^{H}$, for some $i$, then $M^{H_{i}}=M^{H}$ and $U^{H_{i}}=U^{H}$ and there is nothing to prove in this case.)

Let us denote $\operatorname{dim} M^{H}=n$, and let $H_{1}, \ldots, H_{s}$ be ordered in such a way that

(1) $\operatorname{dim} M^{H_{i}}=n-1$, for $1 \leqslant i \leqslant p$,

(2) $\operatorname{dim} M^{H_{i}}=n-2$, for $p+1 \leqslant i \leqslant r$,

(3) $\operatorname{dim} M^{H_{i}} \leqslant n-3$, for $r+1 \leqslant i \leqslant s$. 
Here $0 \leqslant p \leqslant r \leqslant s$. Let us denote

$$
U_{2}=U^{H}-\bigcup_{i=1}^{r} U^{H_{i}}, \quad M_{2}=M^{H}-\bigcup_{i=1}^{r} M^{H_{i}},
$$

and

$$
U_{3}=U^{H}-\bigcup_{i=1}^{s} U^{H_{i}}, \quad M_{3}=M^{H}-\bigcup_{i=1}^{s} M^{H_{i}} .
$$

To prove that "Assertion $H$ " is valid is thus to prove that

$$
j_{\sharp}: \pi_{q}\left(U_{3}\right) \rightarrow \pi_{q}\left(M_{3}\right)
$$

is an isomorphism for every $q \geqslant 0$.

First of all we claim that

$$
i_{\sharp}: \pi_{q}\left(U_{2}\right) \rightarrow \pi_{q}\left(M_{2}\right)
$$

is an isomorphism for all $q \geqslant 0$. If $G$ is nonabelian we have by our assumption (A) that at least one of the following three cases holds.

(i) $p=0$,

(ii) $p=r$,

(iii) $p=1$ and $r=p+1=2$.

In case (i) we have by the assumption in Lemma 4.5, and the fact that in this case $U_{2}$ and $M_{2}$ are connected, that (4) is an isomorphism for every $q \geqslant 0$. In case (ii) it follows by Lemma 4.2 and in case (iii) by Lemma 4.3 that (4) is an isomorphism for all $q \geqslant 0$. (In case $p=r=0$ we have $U_{2}=U^{H}$ and $M_{2}=M^{H}$, and hence (4) is an isomorphism for all q.) If $G$ is abelian it follows by Lemma 4.4 that (4) is an isomorphism for all $q \geqslant 0$.

Since (3) holds it follows, by general position, that the maps

$$
\left\{\begin{array}{l}
i_{\sharp}^{\prime}: \pi_{t}\left(U_{3}\right) \stackrel{\cong}{\rightarrow} \pi_{t}\left(U_{2}\right), \\
i_{\sharp}^{\prime \prime}: \pi_{t}\left(M_{3}\right) \stackrel{\cong}{\rightarrow} \pi_{t}\left(M_{2}\right),
\end{array} \quad t=0,1,\right.
$$

are isomorphisms. Hence using (4) we see that

$$
j_{\#}: \pi_{t}\left(U_{3}\right) \rightarrow \pi_{t}\left(M_{3}\right), \quad t=0,1,
$$

are isomorphisms.

Let $p: \tilde{M}_{2} \rightarrow M_{2}$ be a universal covering of $M_{2}$, i.e., for any component $N$ of $M_{2}$ $p \mid: p^{-1}(N) \rightarrow N$ is a universal covering of the connected manifold $N$. Since $i_{\sharp}: \pi_{t}\left(U_{2}\right) \rightarrow \pi_{t}\left(M_{2}\right)$ is an isomorphism for $t=0$ and 1 it follows that $p \mid: p^{-1}\left(U_{2}\right) \rightarrow$ $U_{2}$ is a universal covering of $U_{2}$. Let us denote $\tilde{U}_{2}=p^{-1}\left(U_{2}\right)$. It follows from (4) that the inclusion $\tilde{i}_{2} \tilde{U}_{2} \rightarrow \tilde{M}_{2}$ induces isomorphisms $\tilde{i}_{\sharp}: \pi_{q}\left(\tilde{U}_{2}\right) \rightarrow \pi_{q}\left(\tilde{M}_{2}\right)$ for all $q \geqslant 0$, and hence

$$
\tilde{i}_{*}: H_{q}\left(\tilde{U}_{2}\right) \rightarrow H_{q}\left(\tilde{M}_{2}\right)
$$

is an isomorphism for every $q \geqslant 0$. 
We have

$$
\begin{gathered}
U_{3}=U_{2}-\bigcup_{k=r+1}^{s}\left(U_{2} \cap U^{H_{k}}\right), \\
M_{3}=M_{2}-\bigcup_{k=r+1}^{s}\left(M_{2} \cap M^{H_{k}}\right),
\end{gathered}
$$

where we in particular have $H_{k} \supsetneq H, k=r+1, \ldots, s$.

Let us now observe that for any subgroup $K$ of $G$ such that $K \supsetneq H$ all the following hold. First of all we have that

$$
\begin{gathered}
U_{2} \cap U^{K}=U^{K}-\bigcup_{i=1}^{r}\left(U^{H_{i}} \cap U^{K}\right)=U^{K}-\bigcup_{i=1}^{r} U^{\left\langle H_{i}, K\right\rangle}, \\
M_{2} \cap M^{K}=M^{K}-\bigcup_{i=1}^{r}\left(M^{H_{i}} \cap M^{K}\right)=M^{K}-\bigcup_{i=1}^{r} M^{\left\langle H_{i}, K\right\rangle} .
\end{gathered}
$$

(Here $\left\langle H_{i}, K\right\rangle$ denotes the subgroup of $G$ generated by $H_{i}$ and $K$.) Since, by our inductive assumption, "Assertion $K$ " holds it follows that

$$
j_{\sharp}: \pi_{q}\left(U_{2} \cap U^{K}\right) \stackrel{\cong}{\rightarrow} \pi_{q}\left(M_{2} \cap M^{K}\right)
$$

is an isomorphism for every $q \geqslant 0$. We consider the coverings $p \mid: p^{-1}\left(U_{2} \cap U^{K}\right) \rightarrow$ $U_{2} \cap U^{K}$ and $p \mid: p^{-1}\left(M_{2} \cap M^{K}\right) \rightarrow M_{2} \cap M^{K}$ and claim that the inclusion $\hat{i}$ induces isomorphisms

$$
\hat{i_{\sharp}}: \pi_{q}\left(p^{-1}\left(U_{2} \cap U^{K}\right)\right) \stackrel{\cong}{\rightarrow} \pi_{q}\left(p^{-1}\left(M_{2} \cap M^{K}\right)\right)
$$

for every $q \geqslant 0$. For any component $N^{\prime}$ of $M_{2} \cap M^{K}$ the homotopy sequences of the coverings $p \mid: p^{-1}\left(U \cap N^{\prime}\right) \rightarrow U \cap N^{\prime}$ and $p \mid: p^{-1}\left(N^{\prime}\right) \rightarrow N^{\prime}$ and the 5-lemma (plus a separate argument in degree 0$)$ together with (9) imply that $\hat{i}_{\sharp}: \pi_{q}\left(p^{-1}\left(U \cap N^{\prime}\right)\right) \rightarrow$ $\pi_{q}\left(p^{-1}\left(N^{\prime}\right)\right)$ is an isomorphism for every $q \geqslant 0$. Hence (10) is an isomorphism for every $q \geqslant 0$ and thus

$$
\hat{i_{*}}: H_{q}\left(p^{-1}\left(U_{2} \cap U^{K}\right)\right) \stackrel{\cong}{\rightarrow} H_{q}\left(p^{-1}\left(M_{2} \cap M^{K}\right)\right)
$$

is an isomorphism for every $q \geqslant 0$.

Furthermore observe that the submanifold $p^{-1}\left(M_{2} \cap M^{K}\right)$ of $\tilde{M}_{2}$ is closed in $\tilde{M}_{2}$, and

$$
\tilde{U}_{2} \cap p^{-1}\left(M_{2} \cap M^{K}\right)=p^{-1}\left(U_{2} \cap U^{K}\right)
$$

The manifold $\tilde{M}_{2}$ is orientable since every component of it is simply connected. Moreover $M_{2} \cap M^{K}$ is orientable since it is an open subset of the contractible manifold $M^{K}$, and hence $p^{-1}\left(M_{2} \cap M^{K}\right)$ is orientable since it is a covering of $M_{2} \cap M^{K}$ (for any component $N$ of $M_{2} \cap M^{K}$, each component of $p^{-1}(N)$ is a connected orientable manifold). Hence

$$
\Re=\left\{p^{-1}\left(M_{2} \cap M^{K}\right) \mid K \supsetneq H\right\}
$$


is a family of orientable closed submanifolds of $\tilde{M}_{2}$ which is closed under intersection. Since (7), (11) and (12) hold we have by Lemma 4.1 and (8) that

$$
j_{*}: H_{q}\left(p^{-1}\left(U_{3}\right)\right) \rightarrow H_{q}\left(p^{-1}\left(M_{3}\right)\right)
$$

is an isomorphism for every $q \geqslant 0$. It follows from (5) that $p \mid: p^{-1}\left(U_{3}\right) \rightarrow U_{3}$ and $p \mid: p^{-1}\left(M_{3}\right) \rightarrow M_{3}$ are universal coverings, i.e., for any components $V_{3}$ of $U_{3}$ and $N_{3}$ of $M_{3}$ we have that $p^{-1}\left(V_{3}\right)$ and $p^{-1}\left(M_{3}\right)$ are simply connected. Hence (13) implies that $j_{\sharp}: \pi_{q}\left(p^{-1}\left(U_{3}\right)\right) \rightarrow \pi_{q}\left(p^{-1}\left(M_{3}\right)\right)$ is an isomorphism for every $q \geqslant 0$, and therefore

$$
j_{\#}: \pi_{q}\left(U_{3}\right) \stackrel{\cong}{\rightarrow} \pi_{q}\left(M_{3}\right)
$$

is an isomorphism for every $q \geqslant 0$. Thus we have proved our claim in (*), and this proves that "Assertion $H$ " is valid. This completes the induction and the proof of Lemma 4.5.

5. Proof of the main results. We are now ready to give the concluding part of the proofs of our main results, and in 5.1 below we prove both Theorems A and B.

THEOREM 5.1. Let $G$ be a finite group acting smoothly on a homotopy sphere $\Sigma^{m}$ such that $\Sigma^{G} \neq \varnothing$ and $\operatorname{dim} \Sigma^{H} \geqslant 5$ for each subgroup $H \neq G$ occurring in $\Sigma$. Assume that either:

(A) Any simultaneous codimension 1 and 2 fixed-point situation is simple, or

(B) $G$ is abelian.

Then the action is topologically equivalent to a linear action if and only if each fixed-point set $\Sigma^{H}$ is homeomorphic to a sphere and each codimension 2 fixed-point situation $\left(\Sigma^{H}, \Sigma^{(>H, 2)}\right)$ is topologically standard.

Proof. The "only if" part is clear as we already noted in the introduction. We shall prove that the conditions are sufficient. First we give the proof in the case when $\operatorname{dim} \Sigma^{G} \geqslant 5$. Let $b \in \Sigma^{G}$ and $M=\Sigma-\{b\}$. Then $M$ is a smooth $G$-manifold, whose underlying smooth manifold is $\mathbf{R}^{m}$, and each fixed-point set $\Sigma^{H}$ is homeomorphic to euclidean space $\mathbf{R}^{n(H)}$, where $n(H)=\operatorname{dim} \Sigma^{H}$. (In fact, in this case, each $\Sigma^{H}$ is diffeomorphic to $\mathbf{R}^{n(H)}$, due to the uniqueness up to diffeomorphism of differentiable structures on $\mathbf{R}^{n}, n \geqslant 5$.)

Let $a \in M^{G}$ and let $U$ be a smooth slice at $a \in M$. We wish to show that the condition in the assumption of Lemma 4.5 is satisfied. Let $H$ be a subgroup of $G$ and $H_{1}, \ldots, H_{r}$ subgroups of $G$ containing $H$ such that $\operatorname{dim} M^{H_{k}}=\operatorname{dim} M^{H}-2,1 \leqslant k$ $\leqslant r$. Our claim is that

$$
i_{\sharp}: \pi_{q}\left(U^{H}-\bigcup_{k=1}^{r} U^{H_{k}}\right) \rightarrow \pi_{q}\left(M^{H}-\bigcup_{k=1}^{r} M^{H_{k}}\right),
$$

where $i$ denotes the inclusion, is an isomorphism for every $q \geqslant 1$. This is seen as follows. Since $\left(\Sigma^{H}, \Sigma^{(>H, 2)}\right)$ is topologically standard it follows that there exists a homeomorphism

$$
\beta:\left(M^{H}, M^{(>H, 2)}\right) \stackrel{\cong}{\rightarrow}\left(\mathbf{R}^{n}, \bigcup_{j=1}^{t} L_{j}^{n-2}\right)
$$


with $\beta(a)=0$, such that if $K \supsetneq H$ and $\operatorname{dim} M^{K}=\operatorname{dim} M^{H}-2$ we have $\beta\left(M^{K}\right)=$ $L_{j_{0}}^{n-2}$, for some $j_{0}$. Here $n=\operatorname{dim} M^{H}$ and each $L_{j}^{n-2}, 1 \leqslant j \leqslant t$, is an $(n-2)$ dimensional linear subspace of $\mathbf{R}^{n}$. (See $\S 1$.) Hence $\beta$ gives a homeomorphism

$$
\beta:\left(M^{H}, \bigcup_{k=1}^{r} M^{H_{k}}\right) \stackrel{\cong}{\rightarrow}\left(\mathbf{R}^{n}, \bigcup_{k=1}^{r} L_{k}^{n-2}\right)
$$

where $\beta\left(M^{H_{k}}\right)=L_{k}^{n-2}, 1 \leqslant k \leqslant r$, and $\beta(a)=0$. It now follows that $a \in M^{H}$ has arbitrarily small neighbourhoods $W$ in $M^{H}$ such that the inclusion

$$
i_{0}: W-\bigcup_{k=1}^{r}\left(W \cap M^{H_{k}}\right) \hookrightarrow M^{H}-\bigcup_{k=1}^{r} M^{H_{k}}
$$

is a homotopy equivalence. Choose such a $W$ with $W \subset U^{H}$. Then we get the inclusion

$$
i^{\prime}: W-\bigcup_{k=1}^{r}\left(W \cap M^{H_{k}}\right) \hookrightarrow U^{H}-\bigcup_{k=1}^{r} U^{H_{k}} .
$$

Let $\alpha: \mathbf{R}^{n}(\rho) \rightarrow U$ be a $G$-homeomorphism. Choose $\varepsilon>0$ such that $\alpha\left(\dot{B}_{\varepsilon}^{n}(\rho)^{H}\right) \subset W$, where $B_{\varepsilon}^{n}(\rho)=\left\{x \in \mathbf{R}^{n}(\rho) \mid\|x\|<\varepsilon\right\}$, and denote $U_{1}=\alpha\left(B_{\varepsilon}^{n}(\rho)\right)$. Then we have the inclusion

$$
i^{\prime \prime}: U_{1}^{H}-\bigcup_{k=1}^{r} U^{H_{k}} \hookrightarrow W-\bigcup_{k=1}^{r}\left(W \cap M^{H_{k}}\right)
$$

The inclusion

$$
i_{1}=i^{\prime} \circ i^{\prime \prime}: U_{1}^{H}-\bigcup_{k=1}^{r} U^{H_{k}} \hookrightarrow U^{H}-\bigcup_{k=1}^{r} U^{H_{k}}
$$

is a homotopy equivalence. Since $\left(i_{1}\right)_{\#}=i_{\#}^{\prime} \circ i_{\#}^{\prime \prime}$ it follows that the inclusion $i^{\prime}$ in (3) induces an epimorphism in homotopy in every degree. Since

$$
\begin{aligned}
\left(i_{0}\right)_{\#} & =i_{\#} \circ i_{\sharp}^{\prime}: \pi_{q}\left(W-\bigcup_{k=1}^{r}\left(W \cap M^{H_{k}}\right)\right) \rightarrow \pi_{q}\left(U-\bigcup_{k=1}^{r} U^{H_{k}}\right) \\
& \rightarrow \pi_{q}\left(M^{H}-\bigcup_{k=1}^{r} M^{M_{k}}\right),
\end{aligned}
$$

and $i_{0}$ is a homotopy equivalence and $i_{\sharp}^{\prime}$ is an epimorphism in every degree it follows that $i_{\sharp}$ is an isomorphism in every degree, i.e., our claim that (1) is an isomorphism for every $q \geqslant 1$ is valid. Hence Lemma 4.5 implies that

$$
j_{\#}: \pi_{q}\left(U_{H}\right) \rightarrow \pi\left(M_{H}\right)
$$

is an isomorphism for every $q \geqslant 0$ and every subgroup $H$ of $G$, i.e., the pair $\left(M_{H}, U_{H}\right)$ is $q$-connected for all $q \geqslant 0$ and every $H$.

We want to apply part II of Proposition 3.2. Since $M$ is a smooth $G$-manifold we have by Theorems 3.6 and 3.8 in [9] that there exists an equivariant triangulation (in fact, smooth) of $M$ which is an equivariant combinatorial manifold. In this way $M$ is given the structure of an equivariant p.1. manifold. Since $M^{G}$ is contractible and we showed above that $U$ satisfies the condition in the assumption of part II of 
Proposition 3.2 , it only remains to prove that $M$ is equivariantly unraveled at infinity. So let $A$ be any compact subset of $M$. Then $G A$ is a compact subset of $M=\Sigma-\{b\}$. Hence there exists a smooth slice $V$ at $b$ in $\Sigma$ such that $V \cap(G A \cup$ $\{a\})=\varnothing$. In particular $V$ is $G$-homeomorphic to some representation space $\mathbf{R}^{m}(\tau)$ for $G$. We claim that $\left(M_{H},(V-\{b\})_{H}\right)$ is 2-connected for every $H$. Let $N=\Sigma-\{a\}$. Then $V \subset N$ and the pair $(N, V)$ is exactly of the same form as the pair $(M, U)$ considered above, and hence the proof given above shows that $\left(N_{H}, V_{H}\right)$ is $q$-connected for all $q \geqslant 0$ and every subgroup $H$. For $H \neq G$ we have

$$
\left(M_{H},(V-\{b\})_{H}\right)=\left(N_{H}, V_{H}\right) \text {, }
$$

so in this case $\left(M_{H},(V-\{b\})_{H}\right)$ is $q$-connected for all $q$ and hence in particular 2-connected. The pair $\left(M_{G},(V-\{b\})_{G}\right)=\left(M^{G},(V-\{b\})^{G}\right)$ is homeomorphic to a pair of the form $\left(S^{k}-\{\infty\}, V^{\prime}-\{\infty\}\right)$, where $V^{\prime}$ is an open neighbourhood of $\infty \in S^{k}$ which is homeomorphic to $\mathbf{R}^{k}$ and $k \geqslant 5$. Hence $\left(M_{G},(V-\{b\})_{G}\right)$ is at least 4-connected and hence in particular 2-connected. Thus $M$ is equivariantly unraveled at infinity. It now follows by Proposition 3.2, part II, that $M$ is $G$-homeomorphic to a representation space $\mathbf{R}^{m}(\rho)$. Since $M=\Sigma-\{b\}$ it follows that the $G$-space $\Sigma$ is $G$-homeomorphic to $S^{m}(\rho \oplus$ id). This completes the proof of Theorem 5.1 in the case when $\operatorname{dim} \Sigma^{G} \geqslant 5$.

In the case when $0 \leqslant \operatorname{dim} \Sigma^{G} \leqslant 4$ the proof is very similar to the above proof. As before we consider the smooth $G$-manifold $M=\Sigma-\{b\}$. By the equivariant tubular neighbourhood theorem (see Bredon [1, Theorem VI.2.2]) there exists a $G$-diffeomorphism $\varphi: E(\eta) \rightarrow U^{\prime}$, where $E(\eta)$ denotes the total space of the normal bundle $\eta$ of $M^{G}$ in $M$ and $U^{\prime}$ is an open $G$-invariant neighbourhood of $M^{G}$ in $M$. Let $k=\operatorname{dim} M^{G}=\operatorname{dim} \Sigma^{G}$. Since $M^{G}$ is homeomorphic to $\mathbf{R}^{k}$, it is in particular contractible and hence, by Corollary 2.5 in Wasserman [18], there exists an isomorphism of smooth $G$-vector bundles $\gamma: M^{G} \times \mathbf{R}^{m-k}\left(\rho^{\prime}\right) \rightarrow E(\eta)$. Let $\omega: \mathbf{R}^{k} \rightarrow M^{G}$ be a homeomorphism. Then

$$
\alpha=\varphi \circ \gamma \circ(\omega \times \mathrm{id}): \mathbf{R}^{k} \times \mathbf{R}^{m-k}\left(\rho^{\prime}\right) \rightarrow U^{\prime}
$$

is a $G$-homeomorphism and $D=\alpha\left(\mathbf{R}^{k} \times B^{m-k}\left(\rho^{\prime}\right)\right)$ is a closed $G$-neighbourhood of $M^{G}$ in $M$. Let

$$
U=\text { Int } D=\alpha\left(\mathbf{R}^{k} \times \stackrel{\circ}{ }^{m-k}\left(\rho^{\prime}\right)\right) .
$$

Now with this choice of the open $G$-subset $U$ the proof proceeds as in the earlier case. Our first claim is that with $U$ defined by (6) the maps in (1) are isomorphisms for all $q \geqslant 1$. The proof of this is almost identical with the one in the earlier case. The set $W \subset U^{H}$ is chosen as before and $\varepsilon>0$ is chosen such that

$$
U_{1}=\alpha\left(\left(\stackrel{\circ}{B}_{\varepsilon}^{k} \times{\stackrel{\circ}{B_{\varepsilon}^{m-k}}}^{m}\left(\rho^{\prime}\right)\right)^{H}\right) \subset W .
$$

Then (2)-(5) is valid and the conclusion is that Lemma 4.5 implies that $\left(M_{H}, U_{H}\right)$ is $q$-connected for all $q \geqslant 0$ and every subgroup $H$. Moreover the inclusion $U_{H} \hookrightarrow D_{H}$ is a homotopy equivalence for every $H$. Hence $\left(M_{H}, D_{H}\right)$ is $q$-connected for all $q \geqslant 0$ and every subgroup $H$. 
It follows that Proposition 3.2, part I, applies if we can show that $M$ is $\mathscr{F}^{\prime}$-equivariantly unraveled at infinity, where $\mathscr{F}^{\prime}=\mathscr{F}-\{G\}$ and $\mathscr{F}$ denotes the family of all subgroups occurring in $M$. But this we already proved when we showed that $\left(M_{H},(V-\{b\})_{H}\right)$ is 2-connected (in fact $q$-connected for all $q \geqslant 0$ ) for every subgroup $H \neq G$. Thus $M$ is $G$-homeomorphic to $\mathbf{R}^{m}(\rho)$, where $\rho=\underbrace{\mathrm{id} \oplus \ldots \oplus \mathrm{id}}_{k} \oplus$ $\rho^{\prime}$, and hence $\Sigma$ is $G$-homeomorphic to $S^{m}(\rho \oplus \mathrm{id})$. This completes the proof of Theorem 5.1.

\section{REFERENCES}

1. G. E. Bredon, Introduction to compact transformation groups, Academic Press, New York, 1972.

2. W. Browder and W. C. Hsiang, Some problems on homotopy theory, manifolds and transformation groups, Proc. Sympos. Pure Math., Vol. 32, Part 2 (Algebraic and Geometric Topology), Amer. Math. Soc., Providence, R. I., 1978, pp. 251-267.

3. M. Brown, The monotone union of $n$-cells is an open n-cell, Proc. Amer. Math. Soc. 12 (1961), $812-814$.

4. J. C. Cantrell, Almost locally flat embedding of $S^{n-1}$ in $S^{n}$, Bull. Amer. Math. Soc. 69 (1963), 716-718.

5. E. H. Connell, D. Montgomery and C. T. Yang, Compact groups in $E^{n}$, Ann. of Math. (2) 80 (1964), 94-103.

6. (Correction to [5]), Ann. of Math. (2) 81 (1965), 194.

7. C. H. Giffen, The generalized Smith conjecture, Amer. J. Math. 88 (1966), 187-198.

8. J. F. P. Hudson, Piecewise linear topology, Benjamin, New York, 1969.

9. S. Illman, Smooth equivariant triangulations of $G$-manifolds for $G$ a finite group, Math. Ann. 233 (1978), 199-220.

10. Approximation of G-maps by maps in equivariant general position and imbeddings of G-complexes, Trans. Amer. Math. Soc. 262 (1980), 113-157.

11. ___ Equivariant engulfing and recognition of linear actions on spheres, Proc. Internat. Conf. on Topology and its Appl., Moscow, 1979 (to appear).

12. M. Rothenberg, Torsion invariants and finite transformation groups, Proc. Sympos. Pure Math., Vol. 32, Part 1 (Algebraic and Geometric Topology), Amer. Math. Soc., Providence, R. I., 1978, pp. 267-311.

13. T. B. Rushing, Topological embeddings, Academic Press, New York, 1973.

14. L. C. Siebenmann, On detecting open collars, Trans. Amer. Math. Soc. 142 (1969), 201-227.

15. E. H. Spanier, Algebraic topology, McGraw-Hill, New York, 1966.

16. J. Stallings, The piecewise-linear structure of euclidean space, Proc. Cambridge Philos. Soc. 58 (1962), 481-488.

17. __ On topologically unknotted spheres, Ann. of Math. (2) 77 (1963), 490-503.

18. A. G. Wasserman, Equivariant differential topology, Topology 8 (1969), 127-150.

19. J. H. C. Whitehead, Simplicial spaces, nuclei and m-groups, Proc. London Math. Soc. (2) 45 (1939), $243-327$.

Department of Mathematics, University of Helsinki, Hallituskatu 15, SF-00100 Helsinki 10 , FINLAND 